\title{
Hadean/Eoarchean Tectonics and Mantle Mixing Induced by Impacts: a Three-dimensional Study
}

\section{Xavier Borgeat ( $\nabla$ xborgeat@student.ethz.ch )}

ETH Zurich: Eidgenossische Technische Hochschule Zurich ～https://orcid.org/0000-0001-5919-4813

\section{Paul Tackley}

ETH Zurich: Eidgenossische Technische Hochschule Zurich https://orcid.org/0000-0003-4878-621X

\section{Research article}

Keywords: Initiation of plate tectonics, Hadean and Eoarchean tectonics, Late Heavy Bombardment, Mixing of Impactor Material, Three-dimensional

Posted Date: November 9th, 2021

DOI: https://doi.org/10.21203/rs.3.rs-1036346/v1

License: (c) (i) This work is licensed under a Creative Commons Attribution 4.0 International License. Read Full License 


\title{
Hadean/Eoarchean tectonics and mantle mixing induced by impacts: A three-dimensional study
}

\author{
Xavier Borgeat ${ }^{*}$ and Paul J. Tackley ${ }^{2}$
}

\begin{abstract}
The timing of the onset of plate tectonics on Earth remains a topic of strong debate, as does the tectonic mode that preceded modern plate tectonics. Understanding possible tectonic modes and transitions between them is also important for other terrestrial planets such as Venus and rocky exoplanets. Recent two-dimensional modelling studies have demonstrated that impacts can initiate subduction during the early stages of terrestrial planet evolution - the Hadean and Eoarchean in Earth's case. Here, we perform three-dimensional simulations of the influence of ongoing multiple impacts on early Earth tectonics and its effect on the distribution of compositional heterogeneity in the mantle, including the distribution of impactor material. We compare two-dimensional and three-dimensional simulations to determine when geometry is important. Results show that impacts can induce subduction in both 2-D and 3-D and thus have a great influence on the tectonic regime. The effect is particularly strong in cases that otherwise display stagnant-lid tectonics: impacts can shift them to having a plate-like regime. In such cases, however, plate-like behaviour is temporary: as the impactor flux decreases the system returns to what it was without impacts. Impacts result in both greater production of oceanic crust and greater recycling of it, increasing the build-up of subducted crust above the core-mantle boundary and in the transition zone. Impactor material is mainly located in the upper mantle, at least at the end of the modelled 500 million year period. In 2-D simulations, in contrast to 3-D simulations, impacts are less frequent but each has a larger effect on surface mobility, making the simulations more stochastic. These stronger 2-D subduction events can mix both recycled basalt and impactor material into the lower mantle. These results thus demonstrate that impacts can make a first-order difference to the early tectonics and mantle mixing of Earth and other large terrestrial planets, and that three-dimensional simulations are important so that effects are not over- or under-predicted.

\section{Keywords}

Initiation of plate tectonics; Hadean and Eoarchean tectonics; Late Heavy Bombardment; Mixing of Impactor Material; Three-dimensional;
\end{abstract}

${ }^{*}$ Correspondence: xborgeat@student.ethz.ch

${ }^{1}$ Department of Earth Sciences, ETH Zurich, Zurich, Switerland

Full list of author information is available at the end of the article

\section{Introduction}

The time of onset of modern-style plate tectonics is still heavily debated. Large uncertainties exist over its 
5 curred before (for reviews see Cawood et al. (2018);

6 Condie (2018); Korenaga (2013); Palin and Santosh

(2020)). Other planets may also undergo changing tectonic mode - of particular interest here is Venus,

which is proposed to have experienced episodic resur-

facing, perhaps caused by global lithospheric overturn/subduction events (e.g. (Armann and Tackley,

2012; Turcotte, 1993)).

The tectonic mode of the early Earth is often described as stagnant lid (e.g. (O'Neill and Debaille, 2014)), with the idea that the lithosphere was basically rigid for a long time until some mechanism managed to break it and initiate plate tectonics (e.g. (Bercovici and Ricard, 2014; Tang et al., 2020)). However, recent works have questioned this view of the Hadean. Hot mantle temperatures would have caused extensive melting and igneous intrusion, as indicated by widespread reworking of the Hadean and Eoarchean crust inferred from zircons (Kirkland et al., 2021), and this intrusion would have weakened the lithosphere and allowed it to deform, accommodating the lateral motion of sections of it. Such behaviour has been demonstrated in both regional models (Fischer and Gerya, 2016; Piccolo et al., 2019; Sizova et al., 2010), where it was named "plume lid" and global models, where it was named "plutonic squishy lid" (Lourenço et al., 2020; Lourenço et al., 2018), occurring for intrusion fractions of $\geq 80 \%$. Thus, while there is general agreement that any subduction that started under early Earth conditions would not result in long-lived subduction zones due to the weakness of the lithosphere (Moyen and Van Hunen, 2012; Sizova et al., 2010; van Hunen and van den Berg, 2008), considerable crustal deformation might well have taken place. This is also consistent with evidence for underthrusting from >4 Gyr zircons (Hopkins et al., 2008). Large plumes might also have had the capability to initiate transient subduction events (Gerya et al., 2015; Pic- ${ }_{42}$ colo et al., 2020).

During that time, the Earth was accreting around ${ }_{44}$ $0.5 \%$ of its current mass, the so-called late veneer, ${ }_{45}$ through impacts. Currently there is much debate over $\quad 46$ whether there was a late peak of impacts, the so-called $\quad 47$ Late Heavy Bombardment (LHB) (e.g. (Bottke and ${ }_{48}$ Norman, 2017; Marchi et al., 2014)), which is proposed 49 to have been caused by a destabilization of the E-belt 50 and asteroid belt caused by a change in Jupiter's or- $\quad 51$ bit (Morbidelli et al., 2012), or whether the impact 52 rate declined monotonically (e.g. (Brasser et al., 2020; 53 Michael et al., 2018; Morbidelli et al., 2018)). In ei- ${ }_{54}$ ther case, the lunar cratering record shows that there 55 was a flux of large impactors extending throughout 56 the Hadean and Eoarchean eons, and thus the Earth 57 should also have been hit by such impactors during 58 this time period.

A number of studies have addressed the influences 60 of impacts on relatively small stagnant lid bodies such $\quad{ }_{61}$ the Moon (Ghods and Arkani-Hamed, 2007; Rolf et al., $\quad 62$ 2017) and Mars (e.g. (Monteux and Arkani-Hamed, ${ }_{63}$ 2014; Reese et al., 2010, 2002; Roberts et al., 2009)), $\quad 64$ but relatively few have studied impacts on larger plan- ${ }_{65}$ etary bodies such as the Earth and Venus, despite their $\quad 66$ potential importance (e.g. (Maruyama et al., 2018)). 67 Gillmann et al. (2016) showed that a large impact 68 can have a substantial influence on the tectonics of 69 a stagnant-lid Venus-like planet, causing an episode 70 of subduction that rolls back from the impact loca- 71 tion. Multiple impacts continuing for some period of ${ }_{72}$ time could thus potentially mobilise the lithosphere for ${ }_{73}$ a corresponding time period. This was first demon- 74 strated by O'Neill et al. (2017), who presented two- 75 dimensional calculations with an impact flux based on 76 Marchi et al. (2014) to show the importance of im- 77 pacts in early Earth tectonics. The importance of such 78 multiple impacts on inducing outgassing on Venus was 79 
demonstrated also using two-dimensional models by Gillmann et al. (2020), although their influence on tectonics was not analysed.

However, impacts may have an exaggerated effect on two-dimensional models because they are effectively infinite in the out-of-plane direction. Thus, we here analyse three-dimensional simulations and compare and contrast them to identical two-dimensional simulations in order to determine whether the same conclusions apply in 3-D. Additionally, we analyse the effect of multiple impacts on mantle compositional structure, focussing on the radial distribution of recycled basaltic crust and on the distribution of impactor material.

\section{Methods Summary}

Full details of the physical and numerical model are given in the later Methods section; here is a brief overview. We couple 3-D or 2-D simulations of mantle convection with a parameterized impact model, with the rate of impacts vs. time based on (Marchi et al., 2014), which is itself based on the sawtooth bombardment time history proposed by Morbidelli et al. (2012), with a clear spike in the impact rate starting $4.1 \mathrm{Gyr}$ ago. This impact history has a lower peak impactor flux that the classical LHB as well as a commonlyused monotonically-decreasing model (see Figure 1 in (Hopkins and Mojzsis, 2015)). We assume a total mass addition of $1.0 * 10^{20} \mathrm{~kg}$, a power-law distribution of impact sizes with an exponent -3.5 and a maximum size of $1000 \mathrm{~km}$ diameter. Impacts are assumed to add heat according to a commonly-used parameterisation; their direct mechanical effects are not modelled here.

Convection and lithospheric dynamics is simulated using StagYY (Tackley, 2000) with assumed physical parameters similar to those in recent papers (Lourenço et al., 2020; Tackley et al., 2013). The model includes strongly temperature-, pressure- and stress-dependent viscosity that combines diffusion creep at low stress with plastic failure at high stress. Physical proper- ties vary with depth under the compressible anelastic ${ }_{118}$ approximation. Compositional variations between the 119 endmembers basalt and harzburgite are included, with $\quad{ }_{120}$ partial melting that can produce basaltic crust. ${ }_{121}$

We simulate a time period of $500 \mathrm{Myr}$, from 4.2 to ${ }_{122}$ 3.7 Gyr before present.

\section{Results}

In this section, we first present the effects of a single impact, and then analyse the long-term evolution of the simulations, contrasting cases with impacts and without impacts, and in 3-D versus 2-D. Both visual aspects and quantitative measures (such as surface mobility and heat flux) are presented. Finally, we focus on compositional aspects, in particular the radial distribution of basaltic material and the radial distribution of impactor material.

\subsection{Effect of an impact}

Figure 1 shows the effect of a $172 \mathrm{~km}$ radius impact in a two-dimensional simulation. The heat addition generates some melt, some of which produces more crust and depleted harzburgitic material beneath. The buoyancy of the hot, depleted, partially molten material produces high stresses and low viscosity, which allows subduction to start at the edge of the impact site. The buoyant material upwells and spreads laterally while the subduction zones roll back. This process occurs very quickly; the second and third columns span only 1 Myr. This sequence of events is in line with previous simulations (Gillmann et al., 2016; O'Neill et al., 2017). In this case, continuous subduction is already taking place (at about 2 o'clock in the images) due to a prior impact $(281 \mathrm{~km}$ radius impactor at a colatitude of 47 degrees). This subduction directly brings lithospheric material, including basaltic crust, into the lower mantle.

Figure 2 shows the effect of a $402.8 \mathrm{~km}$ radius im- ${ }^{153}$ pactor on surface fields of a three-dimensional simula- ${ }^{154}$ 
tion. Two smaller impacts (100 km radius) can be seen in the second and third columns. A cross-section of this major impact is shown in Figure 3. As in 2-D cases, the lateral spreading causes subduction. The majority of the subducted material flows along the $660 \mathrm{~km}$ discontinuity and only a small fraction reaches the lower mantle. Thus, the deeper mantle contains very little basaltic crust and impactor material. In contrast to the two-dimensional simulations, no continuous subduction events lasting for several million years were observed in 3-D. This is the first main difference observed between the two geometries.

\subsection{Long-term evolution}

Simulations with impacts can evolve quite differently from simulations without impacts, as we first explore visually for cases with a yield stress of $55 \mathrm{MPa}$. Figures 4, 5 and 6 show, respectively, the long term evolution of two-dimensional simulations, three-dimensional simulations and cross-sections of three-dimensional simulations. Cases with (top panels) and without (lower panels) impacts are compared and contrasted. Furthermore, two movies are attached showing the timeevolution of 3-D cases.

In two dimensions, the non-impact case displays a stagnant lid over the illustrated time, although there was some subduction earlier on. The basalt field shows a gradually-thickening crust and some basalt accumulation in the transition zone due to the "basalt barrier" mechanism (Davies, 2008; Yan et al., 2020). In contrast, the impacts case shows much evidence of shortlived subduction events, with many slab segments in the lower mantle. As a result, basaltic material accumulates above the core-mantle boundary (CMB) as well as in the transition zone. The crust is thinner than in the no-impact case but there is a thicker layer of depleted material below the crust.

In three dimensions the effect of impacts on tectonics is dramatic (Figure 5). The no-impact case again displays a stagnant lid the entire time, while the im- ${ }^{193}$ pacts case displays considerable tectonic activity. In ${ }^{194}$ the basalt field, circular impact features are clearly ${ }_{195}$ visible while at later times, linear regions of thin crust 196 caused by spreading zones are visible. The crustal ${ }_{197}$ thickness is on average much lower then in the no- ${ }_{198}$ impacts case. The viscosity field displays a complex 199 mixture of low-viscosity impact features and at later 200 times, linear low-viscosity features corresponding to 201 zones of extension or subduction, i.e. tectonic plate ${ }_{202}$ boundaries. Zones of extension are characterized by ${ }_{203}$ higher stress, lower viscosity and a higher tempera- 204 ture.

In the three-dimensional cross-sections (Figure 6), it 206 is clear that the no-impacts case is stagnant lid while 207 in the impacts cases there is substantial subduction, 208 although the lower mantle does not contain as many 209 slab fragments as in the 2-D case. The impacts case ${ }_{210}$ again shows more basalt accumulation above the CMB ${ }_{211}$ and above the $660 \mathrm{~km}$ discontinuity. ${ }_{212}$

\subsection{Effect of the yield stress}

213

The key parameter controlling the tectonic mode in ${ }^{214}$ visco-plastic mantle convection simulations is the yield ${ }_{215}$ stress, as has been much studied in the past (e.g. ${ }^{216}$ (Lourenço et al., 2020; Moresi and Solomatov, 1998; 217 Tackley, 2000)). Here we study the influence of the ${ }_{218}$ yield stress on surface mobility, which is the ratio of ${ }_{219}$ the rms. surface velocity to the volume-averaged rms. 220 velocity (Tackley, 2000). A ratio larger than about 1221 means that the surface is mobile and that subduction 222 is occurring while a value close to zero means that the ${ }^{223}$ surface is immobile, indicating the stagnant-lid regime ${ }_{224}$ (O’Neill et al., 2017; Tackley, 2000).

Figure 7 shows the relationship between the percent- ${ }_{226}$ age of time that the surface is mobile (Mobility $>1$ ) ${ }_{227}$ and the yield stress for cases with both geometries and $\quad 228$ with and without impacts. Simulations without im- 229 pacts display a clear and quite sharp transition from ${ }^{230}$ 
mobile lid (plate) tectonics to a stagnant-lid regime at a yield stress of around $50 \mathrm{MPa}$. In strong contrast, cases with impacts maintain a mobile surface over a much wider range of yield stress (note that the horizontal scale of these graphs is different), with some periods of mobility even at yield stress exceeding 150 MPa. Thus, it is clear that impacts promote surface mobility for yield stress values that would normally cause a stagnant lid regime.

Comparing 2-D and 3-D, the trends are very similar both qualitatively and quantitatively, but there is more stochastic variation (randomness) in 2-D. This is something that is also apparent from other diagnostics discussed later.

Figure 8 shows the surface mobility vs. time for the three different cases in the both dimensions, with and without impacts. The sawtooth bombardment ramps up at 0.1 Gyr. For the lowest yield stress (45 MPa), all cases are generally mobile, although the no-impact cases revert to stagnant lid during for the last $\sim 0.1$ Gyr of the simulations. The higher yield stress cases (85 and $125 \mathrm{MPa}$ ) display a clearer distinction. In these, the no-impact cases are stagnant lid except for an early burst of subduction in the $85 \mathrm{MPa}$ cases. The impact cases, in contrast, are mostly mobile after 0.1 Gyr when the impact-rate sawtooth kicks in. Comparing 2-D and 3-D cases, the 2-D cases display more variability, with mobility sometimes being 0 and sometimes above 2 . The 3 -D cases are also quite timedependent but the mobility is almost never 0 and the peaks are lower than those of the 2-D cases. Particularly in the 85 and $125 \mathrm{MPa} 3-\mathrm{D}$ cases, the mobility gradually decreases with time as the impact rate decreases, approaching 0 by the end of the simulations. This indicates that once the impacts stop, the tectonic mode returns to what it was before the impacts - there is no permanent kick-starting of plate tectonics.
The relationship between periods of surface mobil- ${ }_{268}$ ity and individual large impacts is shown in Figure 9. 269 Spikes in mobility are often associated with impacts, as $\quad 270$ expected. Not all the $\sim 12^{\prime} 000$ impacts occurring dur- ${ }^{271}$ ing the simulations are shown; instead only the largest $\quad 272$ ones as indicated by the right-hand vertical axes. In 273 2 -D, only the impactors that influence the equato- 274 rial annulus, i.e. occur with their own generated heat 275 anomaly of the equator, are shown. Clearly, the num- 276 ber of impacts influencing the 2-D cases is far lower 277 than the total number of impacts in 3-D. On the other ${ }_{278}$ hand, each impact has a stronger influence on the 2-D $\quad 279$ slice than it does in a 3-D domain, causing mobility 280 that lasts for several tens of millions of years. When ${ }_{281}$ there hasn't been an impact for some time, the sys- ${ }_{282}$ tem returns to a stagnant lid state. These two points: ${ }_{283}$ fewer impacts each with more influence, explain the ${ }^{284}$ difference in mobility time series between 2-D and 3-D 285 models.

\subsection{Radial distribution of basalt}

As basaltic crust is produced at the surface, the extent to which basalt is present at various depths in the mantle gives a clear indication of crustal recycling and mantle mixing. Figure 10 shows radial profiles of the azimuthally-averaged basalt fraction of the mantle at the end of each simulation. A composition of 1 is basalt and 0 is harzburgite. All simulations start homogeneous with basalt fraction 0.2.

The compositional profiles of the mantle are similar between all the cases except above the CMB, around the $660 \mathrm{~km}$ discontinuity and near the top. At the $\mathrm{CMB}$ and $660 \mathrm{~km}$ discontinuity, the amount of basalt is dependent on the amount of subducted basalt and thus on the yield stress. The lower the yield stress, the more basalt accumulates above the $\mathrm{CMB}$ and $660 \mathrm{~km}$ discontinuity. 2-D and 3-D cases are somewhat similar but 2-D models display much more basalt accumulation above the CMB. The no-impacts case is also 305 
shown for $85 \mathrm{MPa}$ yield stress. In both geometries, compared to the equivalent impacts case this displays much less basalt accumulation above the CMB. It also displays less depletion (harzburgite) below the crust. Impacts generate melt in this region, thereby depleting it.

The top panels of Figure 11 show the time-evolution of the mean basaltic composition around the $660 \mathrm{~km}$ discontinuity (between 640 and $690 \mathrm{~km}$ depth) over the simulated 0.5 Gyr. The basalt fraction clearly increases with time, even in the no-impact cases. In no-impact cases, dripping/erosion is responsible for removing the base of the crust. Sudden increases and decreases are due to a slab flowing for a while along the discontinuity and then sinking into the lower mantle, which can cause the basaltic composition to drop suddenly. As the yield stress increases and surface mobility decreases, such sudden changes become rarer. The 45 MPa cases indicate that impacts increase crustal recycling and radial mixing even in cases that are anyway mobile lid.

The lower panels of Figure 11 show the time evolution of the mean basalt fraction of the bottom 225 $\mathrm{km}$ of the mantle. The simulations with impacts generally contain more above-CMB basalt that the simulations without impacts, particularly when the noimpact cases are stagnant lid. This is particularly pronounced in the 2-D impact cases with 85 and $125 \mathrm{MPa}$ yield stress. This indicates that impact-induced subduction is more likely to happen in $2 \mathrm{D}$ and that more material is subducted during such an event. Simulations without impacts generally have less basalt in the deep mantle. Sudden increases of the deep mantle basalt fraction are linked to a sudden decrease of the basaltic composition at the $660 \mathrm{~km}$ discontinuity, indicating a causal linkage.
To sum up, the amount of recycled basalt at vari- ${ }_{342}$ ous depths in the mantle is dependent on the surface ${ }^{343}$ mobility and on the geometry of the model. 344

\subsection{Distribution of impactor material}

Whether or not late veneer material was mixed throughout the mantle has implications for geochemical observations. Thus, we track impactor material, starting with the simple assumption that impactor material initially exists at the impactor site. In reality, impactor material might be distributed in a more complex manner that is dependent on the impact angle (e.g. (Golabek et al., 2018)) but that is beyond the scope of the present study.

The radial distribution of impactor material at the end of the simulated period (Figure 12) is different between the 2-D and 3-D models. In 3-D simulations the impactor material resides mostly in the upper mantle with only a small fraction of it reaching the lower mantle, particularly for higher yield stresses, for which a substantial amount of impactor material also stays in the crust. In contrast, in 2-D cases, impactor material is distributed fairly evenly throughout the mantle regardless of yield stress, with even a peak above the CMB. This is consistent with earlier observations that in 2-D, impact-induced subduction events are stronger and easily reach the lower mantle whereas in 3-D they are weaker and tend to get trapped by the $660 \mathrm{~km}$ discontinuity.

The spatial distribution of impactor material is 370 shown in Figure 13, which helps to explain some obser- 371 vations from Figure 12. In 3-D the impactor material 372 is much more prevalent and better mixed in the upper ${ }_{373}$ mantle than the lower mantle, as compared to in 2-D. $\quad 374$ In neither geometry could the material be described 375 as well mixed - the distributions are still very hetero- 376 geneous. In most cases, there is an accumulation of 377 impactor above the $660 \mathrm{~km}$ discontinuity just as there 378 is with subducted crust: the two often travel together. $\quad 379$ 
The bottom right plot $(2-\mathrm{D}, 125 \mathrm{MPa})$ is somewhat anomalous in its distribution of impactor material. In this case the system alternates between stagnant lid and impact-induced subduction, with only few subduction events taking place during the period of simulations, but each subduction event can be bring a lot of surface material into the deep mantle, as seen here: In the left part of the simulations plane a recent subduction event has brought much impactor in the deep mantle.

\subsection{Time series of surface heat flux}

Impacts can also greatly influence the thermal evolution. Here we analyse their influence on the surface heat flux, including both conductive heat flux and eruptive heat flux. Eruptive heat flux, which is the sum of heat loss by cooling of erupted magma from its initial temperature to the surface temperature and latent heat release due to its solidification (Armann and Tackley, 2012; Nakagawa and Tackley, 2012) is a potentially important heat loss mechanism during the Hadean even when impacts are not present (Lourenço et al., 2018; Moore and Webb, 2013; Nakagawa and Tackley, 2012). Eruptive heat flux is directly related to crustal production rate, so we do not separately plot that here.

The conductive heat flux is plotted in the top panels of Figure 14. For non-impact cases, the heat flux is similar in 2-D and 3-D: low (10-30 TW) for the stagnantlid $75 \mathrm{MPa}$ yield stress case and high (about $80 \mathrm{TW}$ ) for the mobile-lid $35 \mathrm{MPa}$ yield stress cases. Impacts greatly increase the conductive heat flux for the cases that would normally be stagnant lid (75 and $125 \mathrm{MPa}$ yield stress). As with other diagnostics considered earlier, the effect is higher and more time-dependent in 2-D cases. Impacts also slightly increase the conductive heat flux for the normally mobile-lid $35 \mathrm{MPa}$ yield stress cases.
Eruptive heat flux (lower panel of Figure 14) shows ${ }_{417}$ similar trends. For non-impact cases it is of similar ${ }_{418}$ magnitude to the conductive heat flux for mobile-lid ${ }_{419}$ (35 MPa) cases but substantially lower for the stagnant $\quad{ }_{420}$ lid $(75 \mathrm{MPa})$ cases. Impacts bring all cases into the ${ }_{421}$ same range as for mobile-lid cases, with much more ${ }_{422}$ variability for the $2-\mathrm{D}$ case. $\quad{ }^{423}$

Higher surface heat flux in the cases with impacts ${ }_{424}$ does not necessarily mean faster mantle cooling be- ${ }_{425}$ cause much of the additional heat is that added by ${ }_{426}$ the impacts, although we do not attempt to decom- ${ }^{427}$ pose this here. At first glance, the low eruptive heat ${ }_{428}$ flux in the stagnant-lid cases (no impacts $75 \mathrm{MPa}{ }_{429}$ seems to contradict previous findings cited above that ${ }_{430}$ eruptive "heat pipe" heat loss could be important in ${ }_{431}$ young stagnant-lid planets, but actually what happens in models of such planets is that the mantle heats up with time because conductive heat loss is less than radiogenic heat addition (as it is here) until magmatic heat loss becomes high enough to prevent further heating up (as in e.g. the simulations of Armann and Tackley (2012)). This heating up does not have time to happen in the $500 \mathrm{Myr}$ simulated here.

\section{Discussion}

A robust finding from the above analyses is that the effects of impacts is different in 2-D models than in 3 -D models. In 2-D models the effect of each impact is larger because the impact is effectively infinite in the out-of-plane direction. Induced subduction is stronger, more likely, and more able to penetrate the $660 \mathrm{~km}$ discontinuity into the lower mantle. Acting against this, the number of major impacts that influences the 2-D equatorial plane is much less than the total number hitting the planet. The net result is that $2-\mathrm{D}$ models display more time-dependence with larger variability in mobility, heat flux and eruption (crustal production) rate, and display more deep recycling of basaltic crust and of impactor material. 
In 3-D, impact-induced subduction tends to be weaker and less material penetrates the $660 \mathrm{~km}$ discontinuity to reach the lower mantle. This has consequences for the radial distribution of impactor material and recycled basaltic crust. The lower mantle is, in 3-D simulations, lacking in these two types of material compared to the two-dimensional simulations. The main consequence is that the impactor material is not as well mixed in 3-D, with a lot of impactor material staying in the upper mantle.

Thus, the choice of model geometry has an influence on the results. A three-dimensional model should be more realistic, giving a better guide as to the influence of impacts on subduction and the possible initiation of plate tectonics. It also gives richer predictions since it predicts two-dimensional surface fields rather than just sections. However, there are many similarities between the results in 2-D and in 3D so if the limitations are borne in mind, two-dimensional models are a useful exploratory tool, allowing one to run more simulations because they run much faster and produce much less data.

In recent years there has been much debate over the time history of impactor flux, with the previously often-accepted idea of a Late Heavy Bombardment (e.g. (Bottke and Norman, 2017; Morbidelli et al., 2012)) losing ground to that of a monotonicallydecreasing impactor flux (e.g. (Brasser et al., 2020; Michael et al., 2018; Morbidelli et al., 2018; Zhu et al., 2019))). Therefore, it is important to consider what a different impactor history might imply for these calculations. Firstly, we note that the peak impact rate of the "Lunar Sawtooth Bombardment" (Morbidelli et al., 2012) assumed here is already lower than that of the classical LHB concept (see Figure 1 in (Hopkins and Mojzsis, 2015)) and that the impact rate coincides with that of the often-used exponential decay model of (Neukum and Ivanov, 1994) after 4.1 Gyr before present (figure 3 in (Morbidelli et al., 2012)). Thus, 493 if the simple exponential decay model was assumed, 494 there would be more impacts during the first $100 \mathrm{Myr} \quad 495$ of the simulation and the same number otherwise. A 496 recent probabilistic estimate of impact flux vs. time ${ }^{497}$ (Brasser et al., 2020) suggests lower impact fluxes by ${ }_{498}$ a factor of several at earlier times. This would result 499 in less frequent induced subduction in the simulations. $\quad 500$

\section{Conclusions}

Here we have examined the influence of Hadean to 502 Eoarchean impacts on subduction and plate tectonics and mantle mixing using three- and two-dimensional numerical models. The simulations show that impacts can have a huge effect on tectonics, inducing subduction and resulting in a higher surface mobility, greater crustal production and greater recycling of crust. In both geometries, impacts can induce subduction in cases that would otherwise have a stagnant lid, facilitating lid mobility over a large range of yield stress values. In such models, subduction is a direct consequence of impacts and after the impact flux dies down, subduction stops and the system returns to being stagnant lid. Thus, impacts do not influence the long-term tectonic mode: they cannot permanently start plate tectonics.

These broad conclusions are consistent with those presented by O'Neill et al. (2017) based on twodimensional models (the paper mentions a 3-D model, but no 3-D results are presented). It is encouraging that similar results are obtained using two completely different numerical codes with somewhat different model setups. For example, O'Neill et al. (2017) included dislocation creep and Peierl's creep, which we do not; while we have a more detailed parameterisation of phase transitions. Furthermore, we analyse three-dimensional models, include melting and crustal production, and study compositional mixing, focussing $\quad{ }_{529}$ 
on the distribution of recycled basaltic crust and of impactor material.

It is important to highlight the differences between the two- and three-dimensional numerical models. Firstly, while the average surface mobility behaves similarly in 2-D and 3-D models, the implications for the deep mantle are quite different. In 2-D models, subduction of basaltic crust and impactor material into the lower mantle occurs over a much wider range of yield stress values in 2 -D compared to 3 -D. In 2 -D, subduction is easier for an impact to trigger, lasts longer and tends to reach the lower mantle, whereas in 3-D, impact-induced subduction is weaker, short-lived and tends to reach only the upper mantle.

Secondly, there is less recycled basaltic crust in the lower mantle in three-dimensional cases than in twodimensional cases with identical parameters. This is linked to the absence of continuous subduction in 3-D. Thirdly, the radial distribution of impactor material differs with geometry. In 2-D, the impactor material spreads throughout the mantle, allowing the mantle to be enriched in HSEs and other elements coming from the impactor material, whereas in 3-D it mainly stays in the upper mantle. This is again a consequence of the absence of continuous subduction in 3D. Thus, the consequences of an impact are more important in 2D.

To sum up, the results show that Hadean and Eoarchean impacts delivering the late veneer are very important for Hadean and Eoarchean tectonics, crustal production and mantle mixing on Earth. In some cases, the addition of impacts can shift the tectonics regime from a stagnant-lid towards a plate-like regime. Such impacts were also likely to have been important on Venus, as was already argued for Venus' outgassing history (Gillmann et al., 2020). Thus, in order to understand the first billion years of Earth's and Venus' history, it is essential to consider impacts.

\section{Methods}

First, we detail the impact model, then the convection model and finally the cases run.

\subsection{Impact History Model}

As discussed earlier, several chronologies for impactor flux versus time have been proposed. Here, for comparison with previous results, we assume the sawtooth-like impact history proposed by Morbidelli et al. (2012) for the Moon. This model predicts a sudden increase in impact rate 4.1 Gyr before present. Before the start of the LHB, Morbidelli et al. (2012) use two different curves to bracket their estimate. The equation for the lower bracket is:

$$
\frac{d N_{20}}{d t}=0.02 * e^{-\left(\frac{4.5-t}{0.01}\right)^{0.5}}
$$

and the equation of the upper bracket is:

$$
\frac{d N_{20}}{d t}=0.025 * e^{-\left(\frac{4.5-t}{0.003}\right)^{0.34}}
$$

After the increase in impactor flux at $4.1 \mathrm{Gyr}$, the ${ }_{583}$ equation becomes:

$$
\frac{d N_{20}}{d t}=2.7 * 10^{-16} * e^{6.93 * t}+5.9 * 10^{-7}
$$

These equations give the number of craters larger than $\quad 586$ $20 \mathrm{~km}$ per $\mathrm{km}^{2}$ per Gyr.

This lunar impact history was re-scaled for the Earth by Marchi et al. (2014). The key parameters are the total mass of LHB impactors and the size distribution. Spatially, impacts are assumed to be equally distributed over Earth's surface. Additionally, a model for the effect of an impact on Earth's interior is necessary, which depends on the velocity and density of the impactor and its impact angle.

\subsubsection{Total mass of impactors}

The total mass accreted by the Earth after initial formation, or "Late Veneer", has been estimated us- 
ing geochemical constraints. Marchi et al. (2014) estimated this mass using highly siderophile elements (HSEs) and found that it lies between $\sim(0.7-3.0) *$ $10^{22} \mathrm{~kg}$, representing up to $0.5 \%$ of Earth's mass. Here we assume that a value at the lower end of this range: a total mass of $1 * 10^{22} \mathrm{~kg}$ (only $0.17 \%$ of Earth's mass), was added by the later stage of bombardment. For simplicity we assume that the density of the impactors is equal to the density of the planet.

\subsubsection{Size distribution of impacts}

We assume a simple power-law

$$
\frac{d N}{d r}=a\left(\frac{r}{r_{0}}\right)^{-\alpha}
$$

with $\alpha=-3.5$, a commonly cited value (de Pater and Lissauer, 2015). For this exponent, most of the mass is delivered by the largest impactors, so the largest impactor size is an important parameter. We assume a largest impactor diameter of $1000 \mathrm{~km}$, roughly the size of Ceres, the largest asteroid, following O'Neill et al. (2017). The smallest impactor diameter is $20 \mathrm{~km}$, arbitrarily chosen in order to avoid having to process a large number of very small impacts that have negligible effect. Given the total mass, size distribution and time history, impacts are generated randomly at each time step. Each simulation has a different random impact history. The normalized size distribution of 30 simulations is shown in Figure 15. Impacts larger than $100 \mathrm{~km}$ radius are rare, therefore the distribution above this size is rather stochastic.

\subsubsection{Spatial distribution of impacts}

It is assumed that each point on Earth's surface has an equal probability of being hit by an impact. Thus, the probability distribution is flat with respect to longitude and sinusoidal with respect to colatitude. Impact locations are generated randomly over the full sphere even for 2-D simulations; the 2-D domain is assumed to be an annulus around the equator and thus only ${ }_{634}$ impacts that are close to this plane will influence it. $\quad{ }_{635}$

\subsection{Effects of an impact}

As in previous studies on the influence of impacts on the mantle, we consider only the thermal effect of an impact, not its mechanical effect such as the formation of craters, ejecta and the redistribution of mass. Our model is based on that of Senshu et al. (2002) and later used by (Monteux et al., 2007) and others (e.g. (Gillmann et al., 2016; Golabek et al., 2011)). An impact creates a shock wave that propagates spherically. Around the impactor position, the pressure rises and is homogeneous, forming an isobaric core.

The ratio, $\gamma_{i c}$, between the radius of the isobaric core $\left(r_{i c}\right)$ and the impactor size $\left(r_{i m p}\right)$ is given by the following formula from Senshu et al. (2002):

$$
\gamma_{i c}=\frac{r_{i c}}{r_{i m p}}=3^{\frac{1}{3}} \approx 1.44
$$

The pressure increase caused by the impact decreases ${ }_{651}$ away from the isobaric core as the square of the distance $r$ from the center of the isobaric core (Monteux et al., 2007). The temperature increase in the isobaric core $\left(T_{0}\right)$ is then given by Monteux et al. (2007):

$$
T_{0}=\frac{4 \pi}{9} \frac{\gamma}{f(m)} \frac{\rho G R^{2}}{C_{p}}
$$

where $\gamma$ is the fraction of kinetic energy converted into thermal energy (around 30\%) and the function $\mathrm{f}(\mathrm{m})$ describe the volume that is heated and is equal to 2.7 in our case. This equation is valid if the impact velocity is equal to the escape velocity. When the ratio $\frac{v_{\text {imp }}}{v_{\text {escape }}}$ is ${ }^{661}$ larger than one the above equation must be multiplied $\quad 662$ by the square of this ratio (Gillmann et al., 2016). Here ${ }_{663}$ we assume an impactor velocity of $25 \mathrm{~km} / \mathrm{s}$, which is 664 2.235 times Earth's escape velocity. So, according to 665 this model, the temperature increase does not depend 666 on the size of the impactor. The temperature increase ${ }_{667}$ decreases as a function of the distance, $r$, from the 668 
center of the isobaric core as given by (Golabek et al., 2011):

$$
T(r)=\Delta T\left(\frac{r_{i c}}{r}\right)^{4.4}
$$

\subsection{Convection Model}

We simulate compressible mantle convection with strongly varying viscosity and partial melting that can produce basaltic crust in either a 3-D spherical shell or a 2-D spherical annulus (Hernlund and Tackley, 2008) (similar to an equatorial slice of the 3-D spherical model) using StagYY (Tackley, 2008). StagYY uses a finite volume method to solves the Stokes equation for velocity and pressure at each time step, and millions of Lagrangian tracers to track composition and melting (Tackley, 2008). It solves the diffusion, viscous dissipation and adiabatic heating terms using finite differences (Tackley, 2008). The boundaries at the surface and at the CMB are free-slip and isothermal, with the core cooling as heat is removed from it.

The model setup and parameters are similar to those assumed in (Lourenço et al., 2020; Tackley et al., 2013). A compressible mantle is assumed with the material properties being pressure-dependent (Tackley et al., 2013) and including the major phase transitions in the olivine and pyroxene-garnet systems. Partial melting may occur when the temperature reaches the solidus, producing basaltic melt, which immediately erupts to form basaltic crust if it is shallower than $300 \mathrm{~km}$ depth. The initial composition of the entire domain is $80 \%$ harzburgite and $20 \%$ basalt, and the local composition evolves with time due to meltinginduced differentiation. Reasonable density profiles are assumed for the basalt and harzburgite end-members, with basalt being denser than harzburgite at most depths but less dense in a region below $660 \mathrm{~km}$, allowing some basalt to be trapped in this region, as pre- viously studied (e.g. (Davies, 2008; Yan et al., 2020)). 705 The rheology is detailed in the next section. 706

Simulations are run for a period of $500 \mathrm{Myr}$, covering $\quad 707$ 4.2 Gyr to 3.7 Gyr before present. The mantle temper- 708 ature is initialised to an adiabat with $1800 \mathrm{~K}$ potential 709 temperature plus $50 \mathrm{~km}$ thick thermal boundary layers $\quad 710$ at top and bottom and random temperature perturba- $\quad 711$ tions of amplitude $20 \mathrm{~K}$. The initial CMB temperature $\quad{ }_{712}$ is $4200 \mathrm{~K}$ and the surface temperature is $300 \mathrm{~K}$. ${ }_{713}$

Additionally, plastic yielding is included in order to 714 failure of the lithosphere, which can results in plate- 715 like behaviour, episodic overturn or stagnant lid tec- 716 tonics depending on the assumed value of the yield ${ }_{717}$ stress (Moresi and Solomatov, 1998; Tackley, 2000). ${ }_{718}$ The yield stress is thus one of main parameters that 719 was changed in the different simulations. Reference vis- $\quad 720$ cosity $\eta_{0}$ was changed in some.

\subsubsection{Viscosity law}

It is assumed that the dominant deformation mechanism is diffusion creep, with plastic failure occurring at higher stresses. An Arrhenius law is used to described the basic temperature- and pressure-dependence of viscosity:

$$
\eta(T, p)_{d i f f}=\eta_{0} \exp \left[\frac{E+p V(p)}{R T}-\frac{E}{R T_{0_{\eta}}}\right]
$$

where $\mathrm{E}$ is the activation energy, $\mathrm{p}$ is the pressure, $\mathrm{T} \quad{ }_{729}$ is the absolute temperature and $\mathrm{V}$ is the activation ${ }_{730}$ volume, which can be pressure-dependent as in Tack- ${ }_{731}$ ley et al. (2013). $\eta_{0}$ is the reference viscosity, which 732 is the viscosity at the reference temperature $T_{0_{\eta}}$ of ${ }_{733}$ $1600 \mathrm{~K}$ and zero pressure. E and V may be different ${ }_{734}$ for each phase. Here we use the rheological parame- ${ }_{735}$ ters in Tackley et al. (2013), in which all upper mantle 736 phases have the same activation parameters based on ${ }_{737}$ Karato and $\mathrm{Wu}$ (1993), all lower mantle phases except $\quad 738$ post-perovskite have the same activation parameters ${ }_{739}$ 
based on Ammann et al. (2009), there is no intrinsic viscosity jump between upper and lower mantles, but a 0.1 viscosity jump going from Bridgmanite to postperovskite, which has activation parameters based on (Ammann et al., 2010).

\subsubsection{Yield stress}

Plastic yielding is assumed, which can lead to plate tectonics-like behaviour (e.g. Moresi and Solomatov (1998); Tackley (2000)). The yield stress has brittle and a ductile components (Schierjott et al., 2020).

$$
\sigma_{y}=\min \left(\sigma_{d u c t}, \sigma_{b r i t t l e}\right)
$$

The brittle yield stress is proportional to pressure with a friction coefficient of 0.5 ,

$$
\sigma_{\text {brittle }}=c_{f} * P
$$

where $c_{f}$ is the Byerlee's law friction coefficient. while the ductile yield stress is given by:

$$
\sigma_{y}=\sigma_{d u c t}+\sigma_{d u c t}^{\prime} p
$$

where $\sigma_{\text {duct }}$ is the surface yield stress (at $\mathrm{p}=0$ ), which is the main parameter varied, and $\sigma_{d u c t}^{\prime}$ is the vertical gradient of the ductile yield stress, assumed to be 0.001 to avoid yielding in the deep mantle. .

The effective viscosity is the minimum of the diffusion creep viscosity and the yielding viscosity:

$$
\eta_{e f f}=\min \left(n_{d i f f}, \frac{\sigma_{y}}{2 \dot{\varepsilon}}\right)
$$

where $\dot{\varepsilon}$ is the second invariant of the strain rate tensor.

The final viscosity value is truncated between $10^{18}$ $\mathrm{Pa} \mathrm{s}$ and $10^{26} \mathrm{~Pa}$ s.

\subsection{Geometries}

3 -D spherical models use the yin-yang grid with 64 radial cells and 192x64x2 azimuthal cells. As a resolution test, one case was repeated with $288 \times 96 \times 2$ azimuthal 770 cells and diagnostics such as the time evolution of mo- 771 bility and radial distribution of basalt were found to be 772 almost the same. The two-dimensional simulations use 773 a full spherical annulus with the same radial resolution $\quad 774$ of 64 cells and a horizontal resolution of 512 cells. Ra- $\quad 775$ dial grid refinement gives higher radial resolution near 776 surface, the $660 \mathrm{~km}$ discontinuity the CMB (Schierjott 777 et al., 2020). This annulus is a 2-D slice from a spher- 778 ical shell domain taken at the equator and neglecting 779 all terms that depend on latitude (Hernlund and Tack- 780 ley, 2008). Some impacts can happen outside the plane ${ }_{781}$ of simulation but still have an effect on the simulation 782 itself. Higher resolution simulations with 1024 x 128783 cells display similar results, indicating that the lower ${ }_{784}$ resolution is sufficient.

\subsection{Cases}

We ran more than 350 two-dimensional simulations ${ }_{787}$ and around 60 three-dimensional simulations, chang- 788 ing mainly the yield stress. Table 1 gives a short ${ }_{789}$ overview of the range of each parameter used for the 790 different simulations.

Table 1 Main parameters of the simulations

\begin{tabular}{|c|c|}
\hline Start time of the simulations & $4.2 \mathrm{Gyr}$ \\
Start time of the impacts & $4.1 \mathrm{Gyr}$ \\
Duration of the simulations & $0.5 \mathrm{Gyr}$ \\
Reference viscosity, $\eta_{0}$ & $1.0 \mathrm{e} 20 \mathrm{~Pa} \mathrm{~s}$ \\
Yield Stress & $35-245 \mathrm{MPa}$ \\
Dimensions & $2-\mathrm{D} \mathrm{\&} \mathrm{3-D}$ \\
Initial Temperature & $1800 \mathrm{~K}$ \\
Surface Temperature & $300 \mathrm{~K}$ \\
CMB Temperature & $4200 \mathrm{~K}$ \\
\hline
\end{tabular}

The simulations ran on the ETH Euler cluster on 792 32 cores for two-dimensional simulations and on 64793 cores for the three-dimensional simulations.

Abbreviations 72 ,

7

5

政

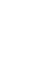

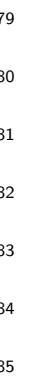




\section{Availability of data and material}

The datasets used and/or analysed during the current study are available

from the corresponding author on reasonable request.

\section{Competing interests}

The authors declare that they have no competing interest.

\section{Funding}

Simulations were run on shares of the Euler cluster bought by funding from the ETH Scientific Equipment Commission. Recent developments of StagYY have benefitted from funding from the ETH/CSCS Platform for Advanced Scientific Computing (PASC) program.

\section{Authors' contributions}

PJT proposed the topic, conceived and designed the study. XB carried out the experimental study, analyzed the data and wrote the first manuscript draft. PJT helped in the interpretation and in writing the final manuscript. Both authors have read and approved the final manuscript.

\section{Authors' information}

$\mathrm{XB}$ is studying for a Masters Degree in Earth Sciences at ETH Zurich and performed this study as his Bachelors thesis. PJT has been a professor in the Institute of Geophysics at ETH Zurich since 2005 and has studied mantle convection using numerical simulations since the early 1990 s.

\section{Acknowledgements}

We thank Antoine Rozel for being a co-examiner on XB's Bachelor's thesis and the ETH SEC and CSCS/PASC for their financial support.

\section{Author details}

${ }^{1}$ Department of Earth Sciences, ETH Zurich, Zurich, Switerland. ${ }^{2}$ Institute of Geophysics, Department of Earth Sciences, ETH Zurich, Zurich, Switzerland.

\section{References}

Ammann, M.W, Brodholt, J.P, Dobson, D.P (2009) Dft study of migration enthalpies in mgsio3 perovskite. Phys. Chem. Minerals 36, 151-158. doi:10.1007/s00269-008-0265-z

Ammann, M.W, Brodholt, J.P, Wookey, J, Dobson, D.P (2010) First-principles constraints on diffusion in lower-mantle minerals and a weak d" layer. Nature 465, 462-465

Armann, M, Tackley, P.J (2012) Simulating the thermo-chemical magmatic and tectonic evolution of venus' mantle and lithosphere: two-dimensional models. J. Geophys. Res. 117 12003-1010292012004231. doi:10.1029/2012JE004231

Bercovici, D, Ricard, Y (2014) Plate tectonics, damage and inheritance. Nature 508(7497), 513-6. doi:10.1038/nature13072

Bottke, W.F, Norman, M.D (2017) The late heavy bombardment. Annual Review of Earth and Planetary Sciences 45(1), 619-647. doi:10.1146/annurev-earth-063016-020131

Brasser, R, Werner, S.C, Mojzsis, S.J (2020) Impact bombardment chronology of the terrestrial planets from 4.5 ga to 3.5 ga. Icarus 338. doi:10.1016/j.icarus.2019.113514

Cawood, P.A, Hawkesworth, C.J, Pisarevsky, S.A, Dhuime, B, Capitanio, F.A, Nebel, O (2018) Geological archive of the onset of plate tectonics. Philos Trans A Math Phys Eng Sci 376(2132). doi:10.1098/rsta.2017.0405

Condie, K.C (2018) A planet in transition: The onset of plate tectonics on earth between 3 and 2 ga? Geoscience Frontiers 9(1), 51-60. doi:10.1016/j.gsf.2016.09.001

Davies, G.F (2008) Episodic layering of the early mantle by the 'basalt barrier' mechanism. Earth Planet. Sci. Lett. 275, 382-392

de Pater, I, Lissauer, J.J (2015) Planetary Sciences, 2nd edn. Cambridge University Press, ??? doi:10.1017/CBO9781316165270

Fischer, R, Gerya, T (2016) Early earth plume-lid tectonics: A high-resolution $3 \mathrm{~d}$ numerical modelling approach. Journal of Geodynamics 100, 198-214. doi:10.1016/j.jog.2016.03.004

Gerya, T.V, Stern, R.J, Baes, M, Sobolev, S.V, Whattam, S.A (2015) Plate tectonics on the earth triggered by plume-induced subduction initiation. Nature 527(7577), 221-225

Ghods, A, Arkani-Hamed, J (2007) Impact-induced convection as the main mechanism for formation of lunar mare basalts. J. Geophys. Res. 112(E3), 03005. doi:10.1029/2006je002709

Gillmann, C, Golabek, G.J, Raymond, S.N, Schönbächler, M, Tackley, P.J, Dehant, V, Debaille, V (2020) Dry late accretion inferred from venus's coupled atmosphere and internal evolution. Nature Geoscience 13(4), 265-269. doi:10.1038/s41561-020-0561-x

Gillmann, C, Golabek, G.J, Tackley, P.J (2016) Effect of a single large impact on the coupled atmosphere-interior evolution of venus. Icarus 268, 295-312

Golabek, G.J, Emsenhuber, A, Jutzi, M, Asphaug, E.I, Gerya, T.V (2018) Coupling sph and thermochemical models of planets: Methodology and example of a mars-sized body. Icarus 301, 235-246. doi:10.1016/j.icarus.2017.10.003

Golabek, G.J, Keller, T, Gerya, T.V, Zhu, G, Tackley, P.J, Connolly, J.A (2011) Origin of the martian dichotomy and tharsis from a giant impact causing massive magmatism. Icarus 215(1), 346-357

Hernlund, J.W, Tackley, P.J (2008) Modeling mantle convection in the spherical annulus. Physics of the Earth and Planetary Interiors 171(1-4), 48-54

Hopkins, M, Harrison, T.M, Manning, C.E (2008) Low heat flow inferred from ¿4 gyr zircons suggests hadean plate boundary interactions. Nature 456(7221), 493-6. doi:10.1038/nature07465

Hopkins, M, Mojzsis, S.J (2015) A protracted timeline for lunar bombardment from mineral chemistry, ti thermometry and $\mathrm{u}-\mathrm{pb}$ geochronology of apollo 14 melt breccia zircons. Contributions to Mineralogy and Petrology 169(3), 30

Karato, S, Wu, P (1993) Rheology of the upper mantle - a synthesis. Science 260(5109), 771-778

Kirkland, C.L, Hartnady, M.I.H, Barham, M, Olierook, H.K.H, Steenfelt, A, Hollis, J.A (2021) Widespread reworking of hadean-to-eoarchean continents during earth's thermal peak. Nat Commun 12(1), 331. doi:10.1038/s41467-020-20514-4

Korenaga, J (2013) Initiation and evolution of plate tectonics on earth: theories and observations. Annual review of earth and planetary sciences $41,117-151$ 
Lourenço, D.L, Rozel, A.B, Ballmer, M.D, Tackley, P.J (2020)

Plutonic-squishy lid: A new global tectonic regime generated by intrusive magmatism on earth-like planets. Geochemistry, Geophysics, Geosystems 21(4), 2019-008756

Lourenço, D.L, Rozel, A.B, Gerya, T, Tackley, P.J (2018) Efficient cooling of rocky planets by intrusive magmatism. Nature Geoscience 11(5), 322-327. doi:10.1038/s41561-018-0094-8

Marchi, S, Bottke, W, Elkins-Tanton, L, Bierhaus, M, Wuennemann, K, Morbidelli, A, Kring, D (2014) Widespread mixing and burial of earth's hadean crust by asteroid impacts. Nature 511(7511), 578-582

Maruyama, S, Santosh, M, Azuma, S (2018) Initiation of plate tectonics in the hadean: Eclogitization triggered by the abel bombardment. Geoscience Frontiers 9(4), 1033-1048

Michael, G, Basilevsky, A, Neukum, G (2018) On the history of the early meteoritic bombardment of the moon: Was there a terminal lunar cataclysm? Icarus 302, 80-103. doi:10.1016/j.icarus.2017.10.046

Monteux, J, Arkani-Hamed, J (2014) Consequences of giant impacts in early mars: Core merging and martian dynamo evolution. Journal of Geophysical Research: Planets 119(3), 480-505. doi:10.1002/2013je004587

Monteux, J, Coltice, N, Dubuffet, F, Ricard, Y (2007)

Thermo-mechanical adjustment after impacts during planetary growth. Geophysical Research Letters 34(24)

Moore, W.B, Webb, A.A.G (2013) Heat-pipe earth. Nature 501(7468), 501-505. doi:10.1038/nature12473

Morbidelli, A, Nesvorny, D, Laurenz, V, Marchi, S, Rubie, D.C, Elkins-Tanton, L, Wieczorek, M, Jacobson, S (2018) The timeline of the lunar bombardment: Revisited. Icarus 305, 262-276. doi:10.1016/j.icarus.2017.12.046

Morbidelli, A, Marchi, S, Bottke, W.F, Kring, D.A (2012) A sawtooth-like timeline for the first billion years of lunar bombardment. Earth and Planetary Science Letters 355, 144-151

Moresi, L, Solomatov, V (1998) Mantle convection with a brittle lithosphere - thoughts on the global tectonic styles of the earth and venus. Geophys. J. Int. 133(3), 669-682

Moyen, J-F, Van Hunen, J (2012) Short-term episodicity of archaean plate tectonics. Geology 40(5), 451-454

Nakagawa, T, Tackley, P.J (2012) Influence of magmatism on mantle cooling, surface heat flow and urey ratio. Earth Planet. Sci. Lett. 329-330, 1-10. doi:10.1016/j.epsl.2012.02.011.

Neukum, G, Ivanov, B.A (1994) Crater size distributions and impact probabilities on earth from lunar, terrestrial-planet, and asteroid cratering data. In: Gehrels, T. (Ed.), Hazards Due to Comets and Asteroids, University of Arizona Press, Tucson, AZ, USA, 359-416

O'Neill, C, Debaille, V (2014) The evolution of hadean-eoarchaean geodynamics. Earth and Planetary Science Letters 406, 49-58

O'Neill, C, Marchi, S, Zhang, S, Bottke, W (2017) Impact-driven subduction on the hadean earth. Nature Geoscience 10(10), 793-797

Palin, R.M, Santosh, M (2020) Plate tectonics: What, where, why, and when? Gondwana Research. doi:10.1016/j.gr.2020.11.001

Piccolo, A, Palin, R.M, Kaus, B.J.P, White, R.W (2019) Generation of earth's early continents from a relatively cool archean mantle. Geochemistry, Geophysics, Geosystems 20(4), 1679-1697. doi:10.1029/2018gc008079

Piccolo, A, Kaus, B.J.P, White, R.W, Palin, R.M, Reuber, G.S (2020) Plume - lid interactions during the archean and implications for the generation of early continental terranes. Gondwana Research 88, 150-168. doi:10.1016/j.gr.2020.06.024

Reese, C.C, Orth, C.P, Solomatov, V.S (2010) Impact origin for the martian crustal dichotomy: Half-emptied or half filled. J. Geophys. Res. submitted

Reese, C.C, Solomatov, V.S, Baumgardner, J.R (2002) Survival of impact-induced thermal anomalies in the martian mantle. Journal of Geophysical Research-Planets 107(E10), 5082

Roberts, J.H, Lillis, R.J, Manga, M (2009) Giant impacts on early mars and the cessation of the martian dynamo. Journal of Geophysical Research 114(E4). doi:10.1029/2008je003287

Rolf, T, Zhu, M.H, Wünnemann, K, Werner, S.C (2017) The role of impact bombardment history in lunar evolution. Icarus 286 138-152. doi:10.1016/j.icarus.2016.10.007

Schierjott, J, Rozel, A, Tackley, P (2020) On the self-regulating effect of grain size evolution in mantle convection models: application to thermochemical piles. Solid Earth 11(3), 959-982

Senshu, H, Kuramoto, K, Matsui, T (2002) Thermal evolution of a growing mars. Journal of Geophysical Research: Planets 107(E12), 1-1

Sizova, E, Gerya, T, Brown, M, Perchuk, L.L (2010) Subduction styles in the precambrian: Insight from numerical experiments. Lithos 116(3-4), 209-229

Tackley, P.J (2000) Self-consistent generation of tectonic plates in time-dependent, three-dimensional mantle convection simulations. Geochemistry, Geophysics, Geosystems 1(8)

Tackley, P.J (2008) Modelling compressible mantle convection with large viscosity contrasts in a three-dimensional spherical shell using the yin-yang grid. Physics of the Earth and Planetary Interiors 171(1-4), 7-18

Tackley, P.J, Ammann, M, Brodholt, J.P, Dobson, D.P, Valencia, D (2013) Mantle dynamics in super-earths: Post-perovskite rheology and self-regulation of viscosity. Icarus 225(1), 50-61

Tang, C.A, Webb, A.A.G, Moore, W.B, Wang, Y.Y, Ma, T.H, Chen, T.T (2020) Breaking earth's shell into a global plate network. Nat Commun 11(1), 3621. doi:10.1038/s41467-020-17480-2

Turcotte, D.L (1993) An episodic hypothesis for venusian tectonics. J. Geophys. Res. 98(E9), 17061-17068

van Hunen, J, van den Berg, A.P (2008) Plate tectonics on the early earth: Limitations imposed by strength and buoyancy of subducted lithosphere. Lithos 103(1-2), 217-235. doi:10.1016/j.lithos.2007.09.016

Yan, J, Ballmer, M.D, Tackley, P.J (2020) The evolution and distribution of recycled oceanic crust in the earth's mantle: Insight from 
geodynamic models. Earth and Planetary Science Letters 537. doi:10.1016/j.epsl.2020.116171

Zhu, M.H, Artemieva, N, Morbidelli, A, Yin, Q.Z, Becker, H, Wunnemann, K (2019) Reconstructing the late-accretion history of the moon. Nature 571(7764), 226-229. doi:10.1038/s41586-019-1359-0
Figure legends

Animation showing the long-term evolution of three-dimensional simulations with and without impacts from Figure 5 . The basalt fraction field is plotted.

\section{Animation 2}

Animation showing 400 Myr evolution of a three-dimensional simulation with

impacts and a yield stress of $45 \mathrm{MPa}$, showing temperature, strain rate and basalt at the surface and a cross-section showing the distribution of impactor material in the mantle.
1011

1012 


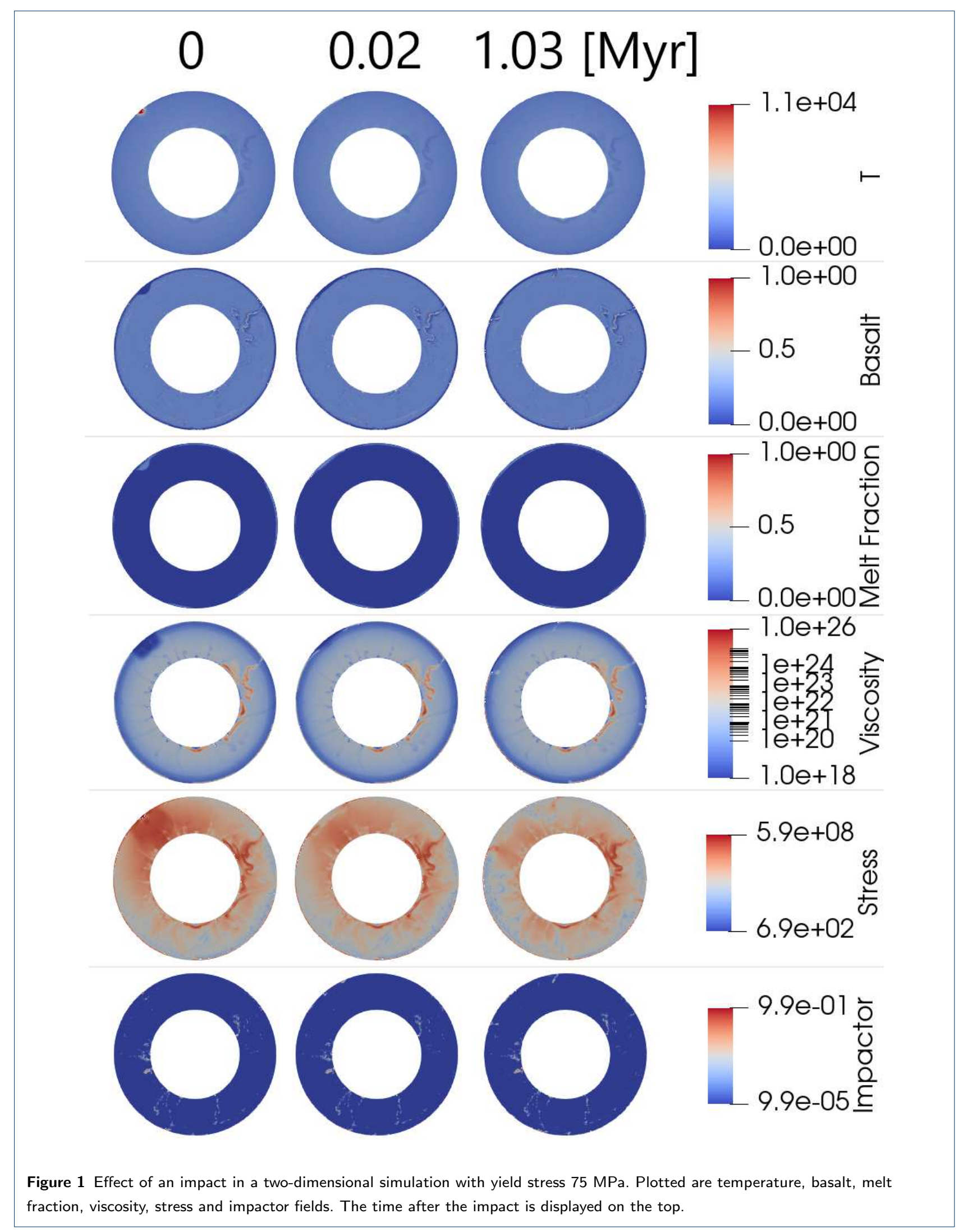




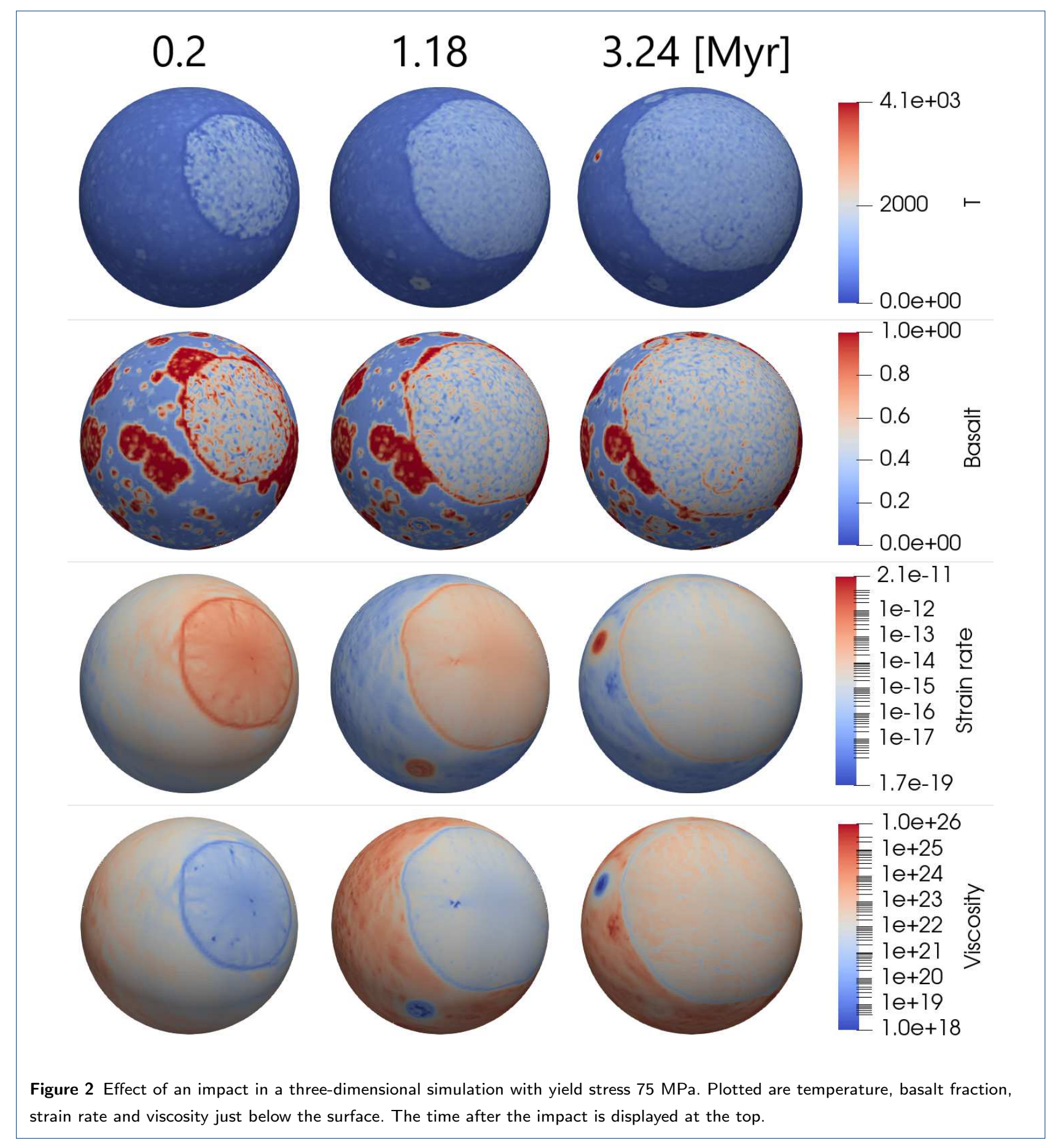




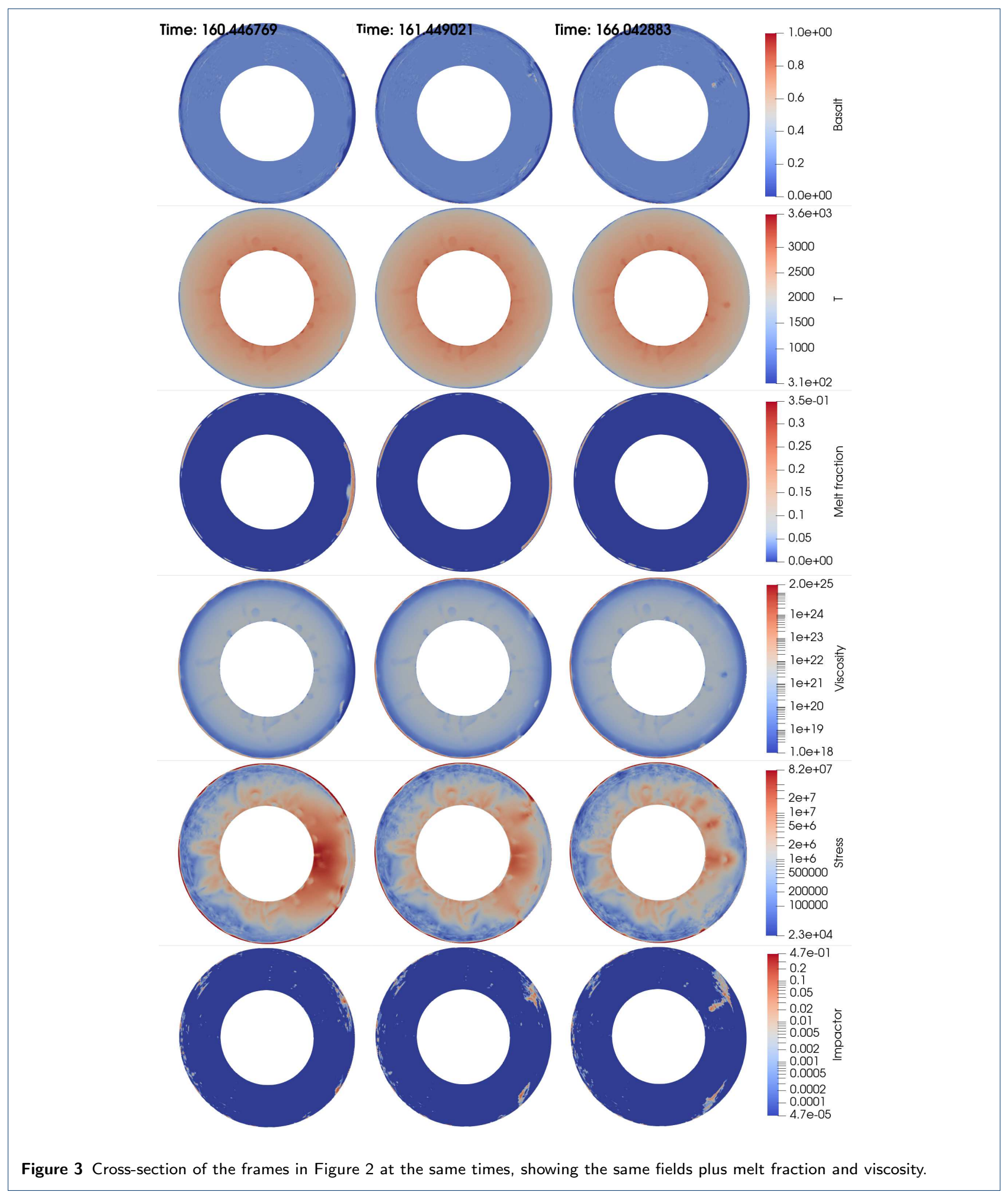




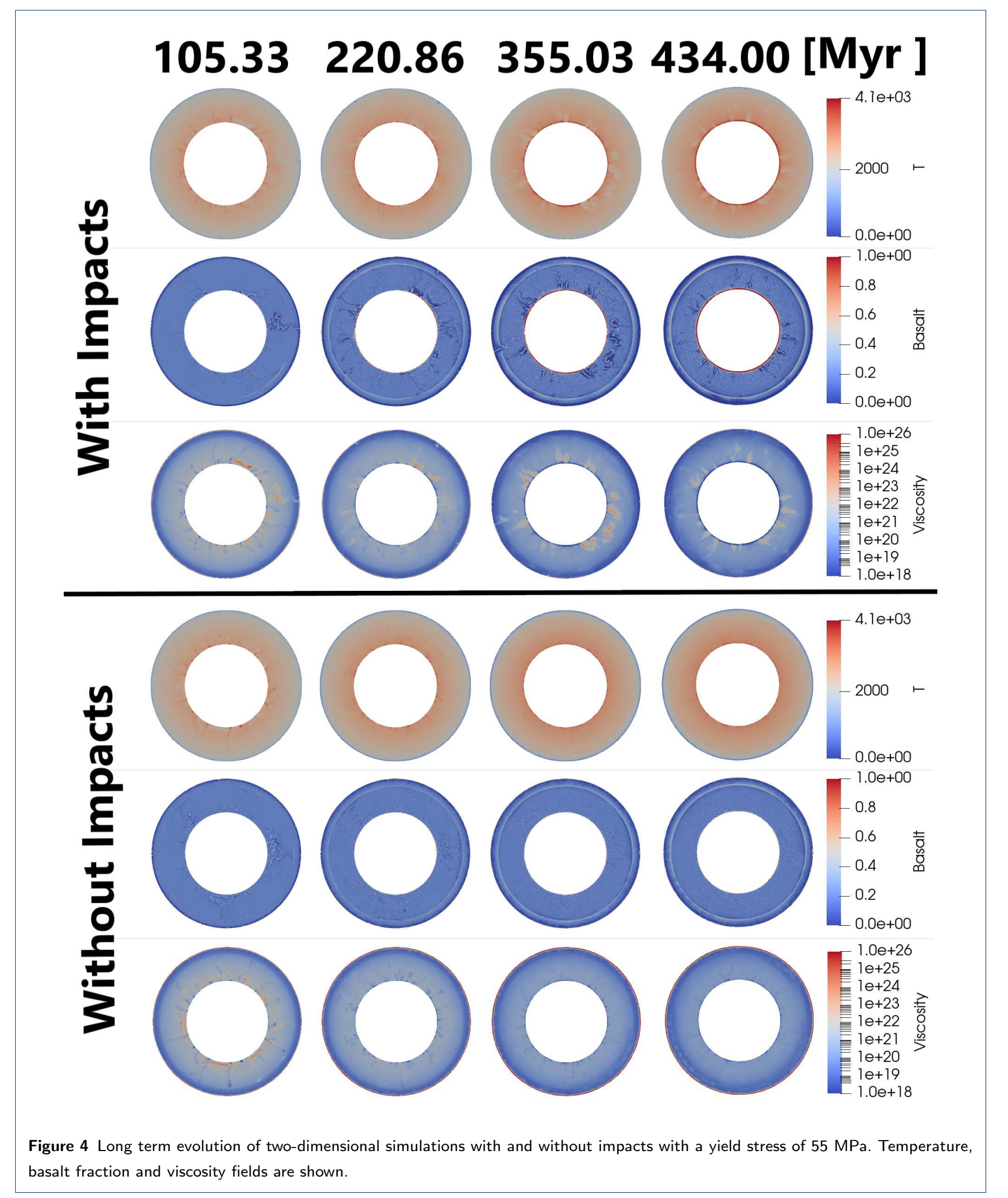




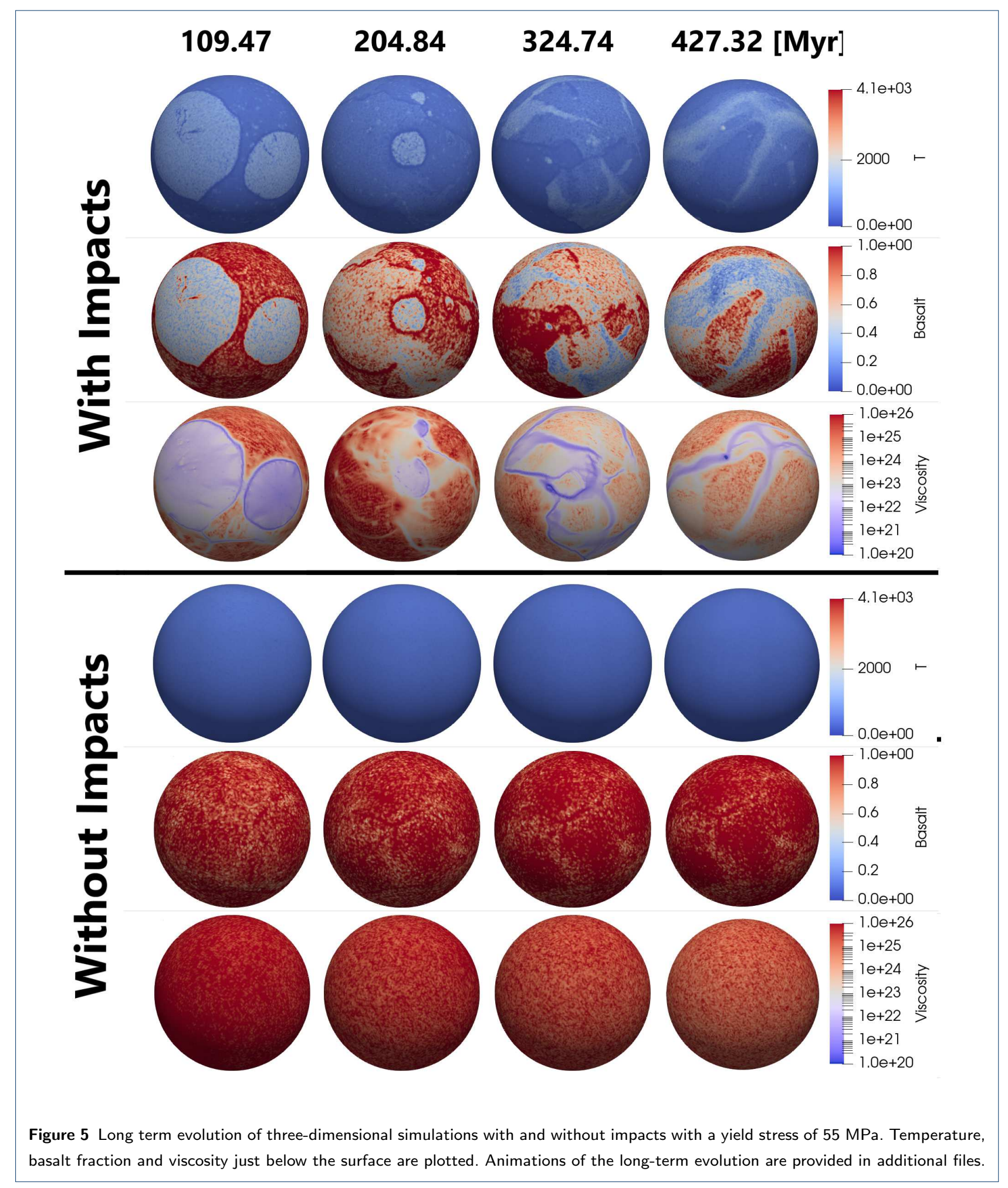




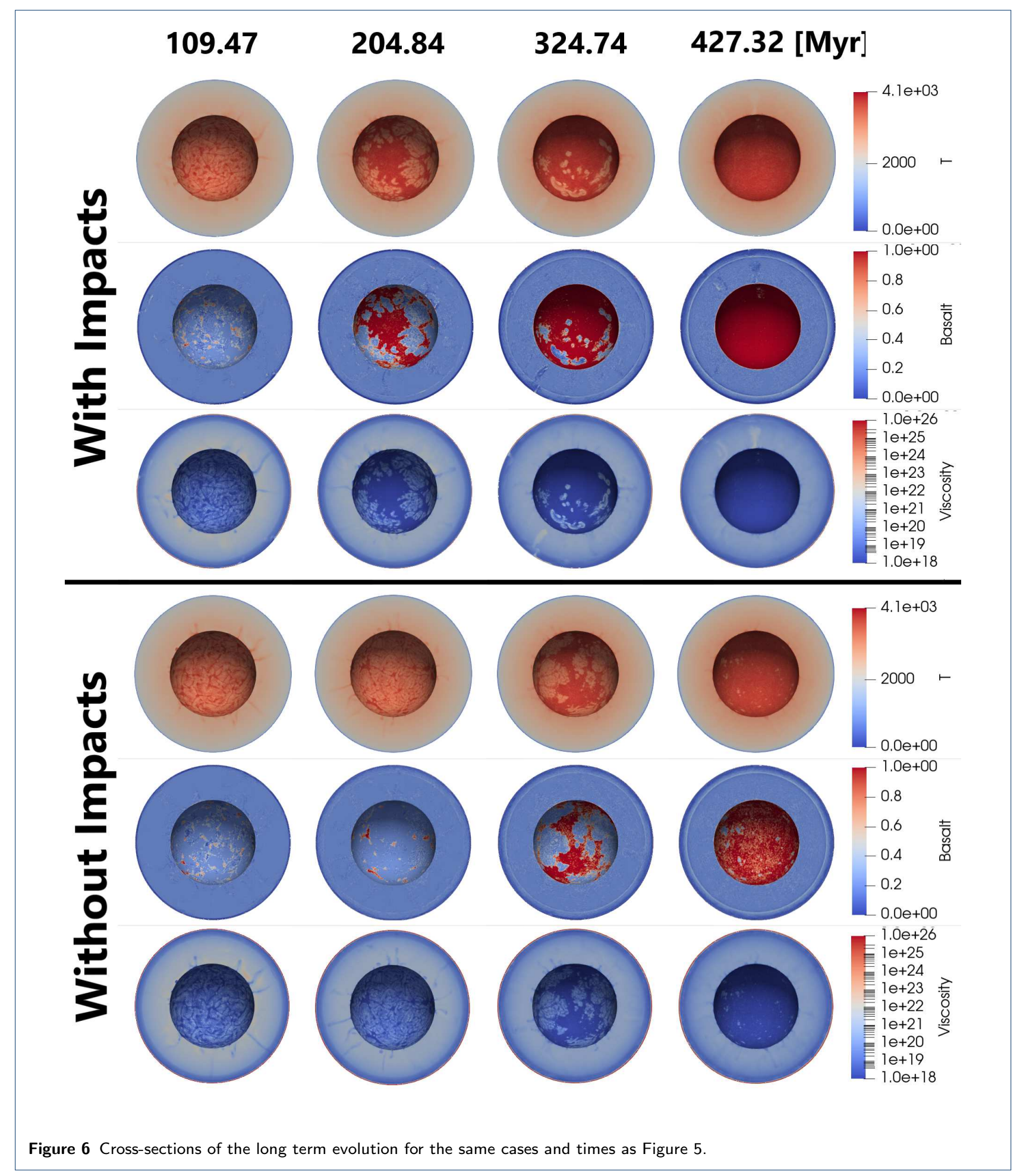



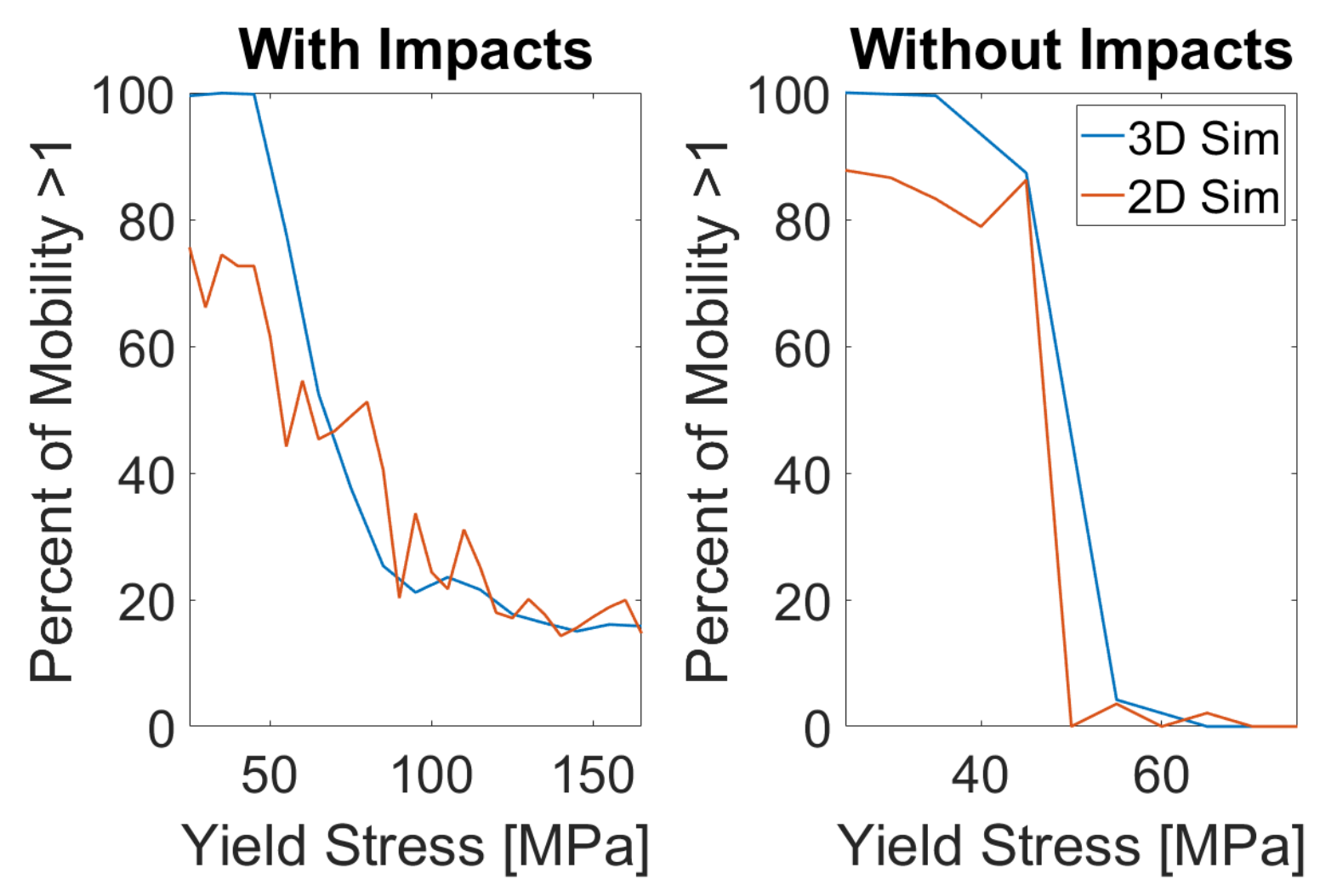

Figure 7 Percent of time when the surface mobility is higher than 1 versus yield stress for two- and three-dimensional cases with and without impacts.

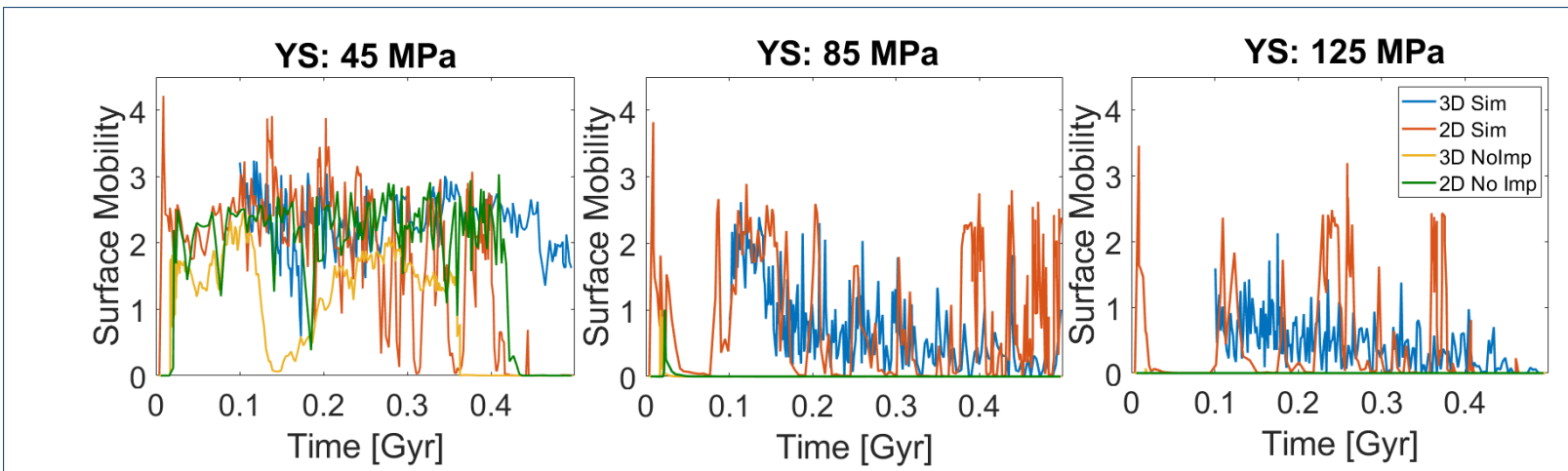

Figure 8 Surface mobility versus time for 2-D and 3-D cases with and without impacts for three different yield stress values. 

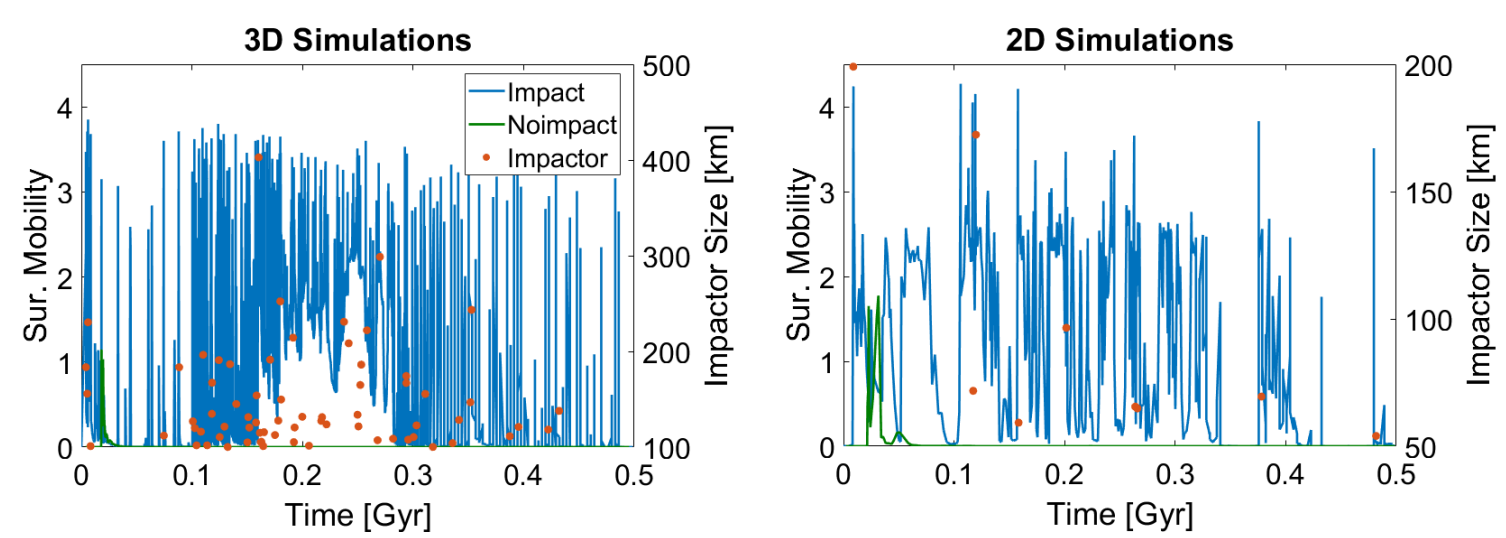

Figure 9 Surface mobility and impactor size versus time for cases with yield stress $75 \mathrm{MPa}$ in 2-D and 3-D. Only the larger impacts are shown as labelled on the right-hand vertical axis. In 2-D only impacts generating a thermal anomaly that intersects the simulation plane are plotted. The simulations with impacts are the same used to show the effect of an impact (Figures 1,2 and 3 ).

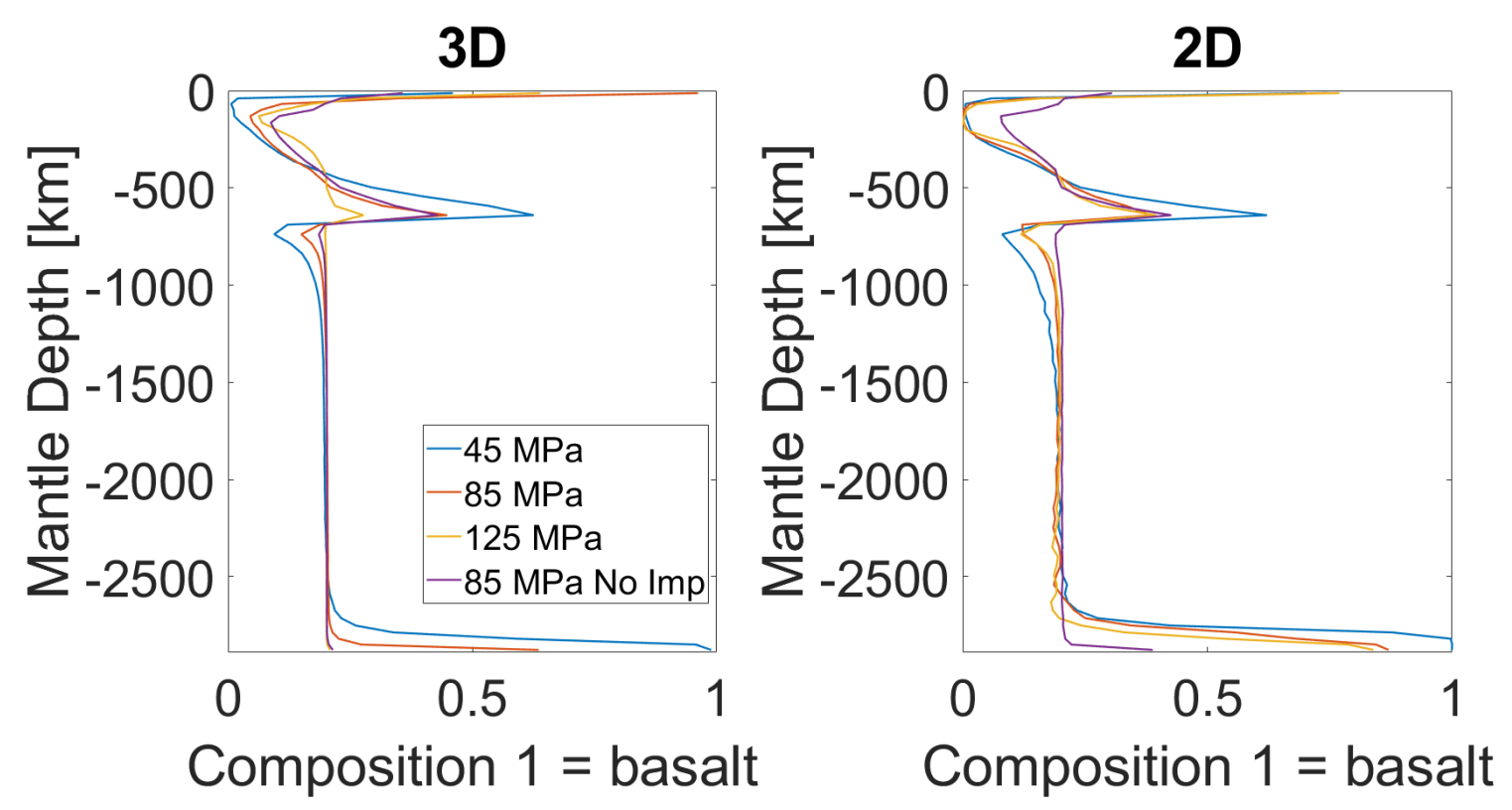

Figure 10 Azimuthally-averaged basalt fraction versus radius at the end of the simulated time for various yield stresses in 2-D and 3-D. A composition of 1 is basalt while a composition of 0 is harzburgite. 

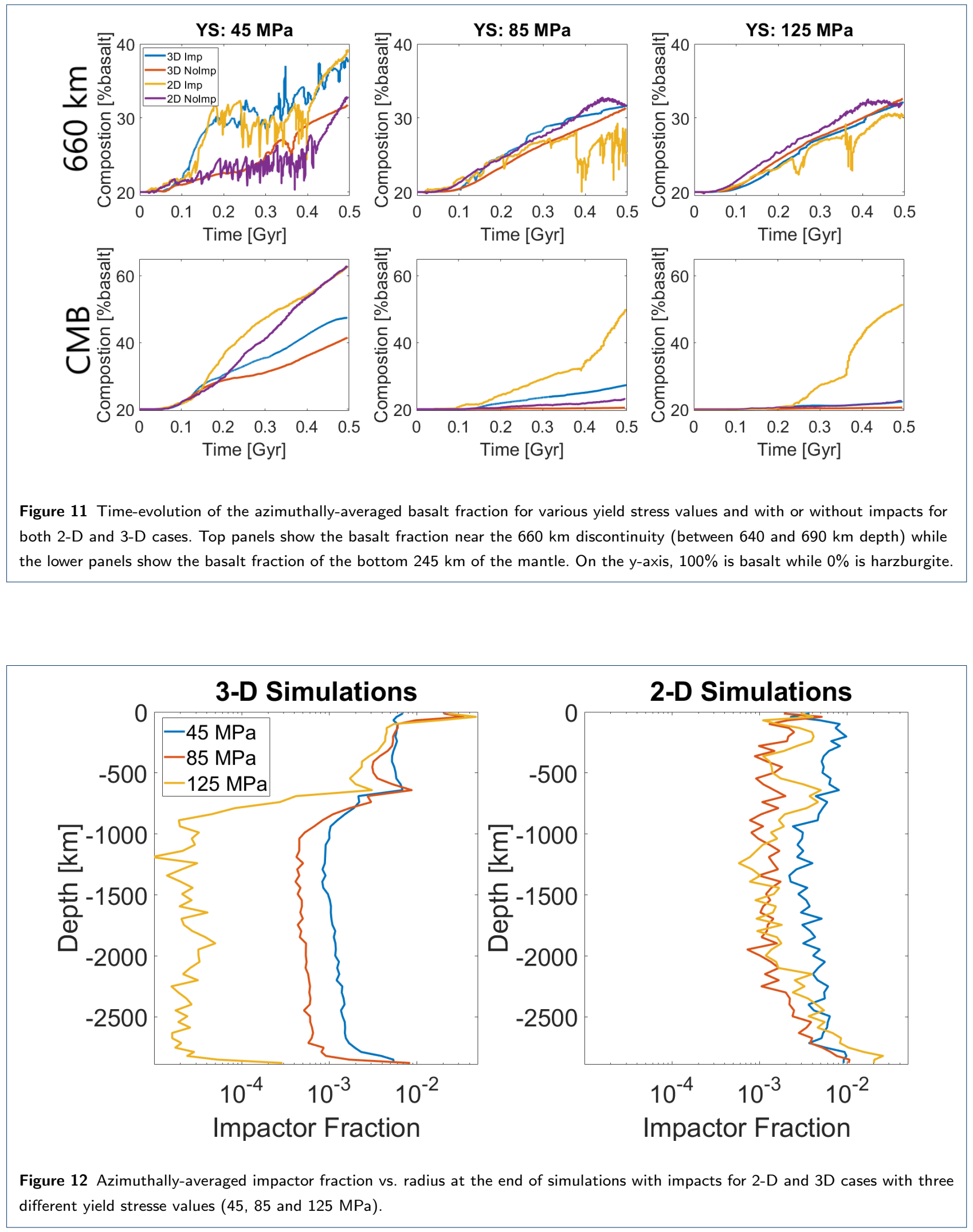


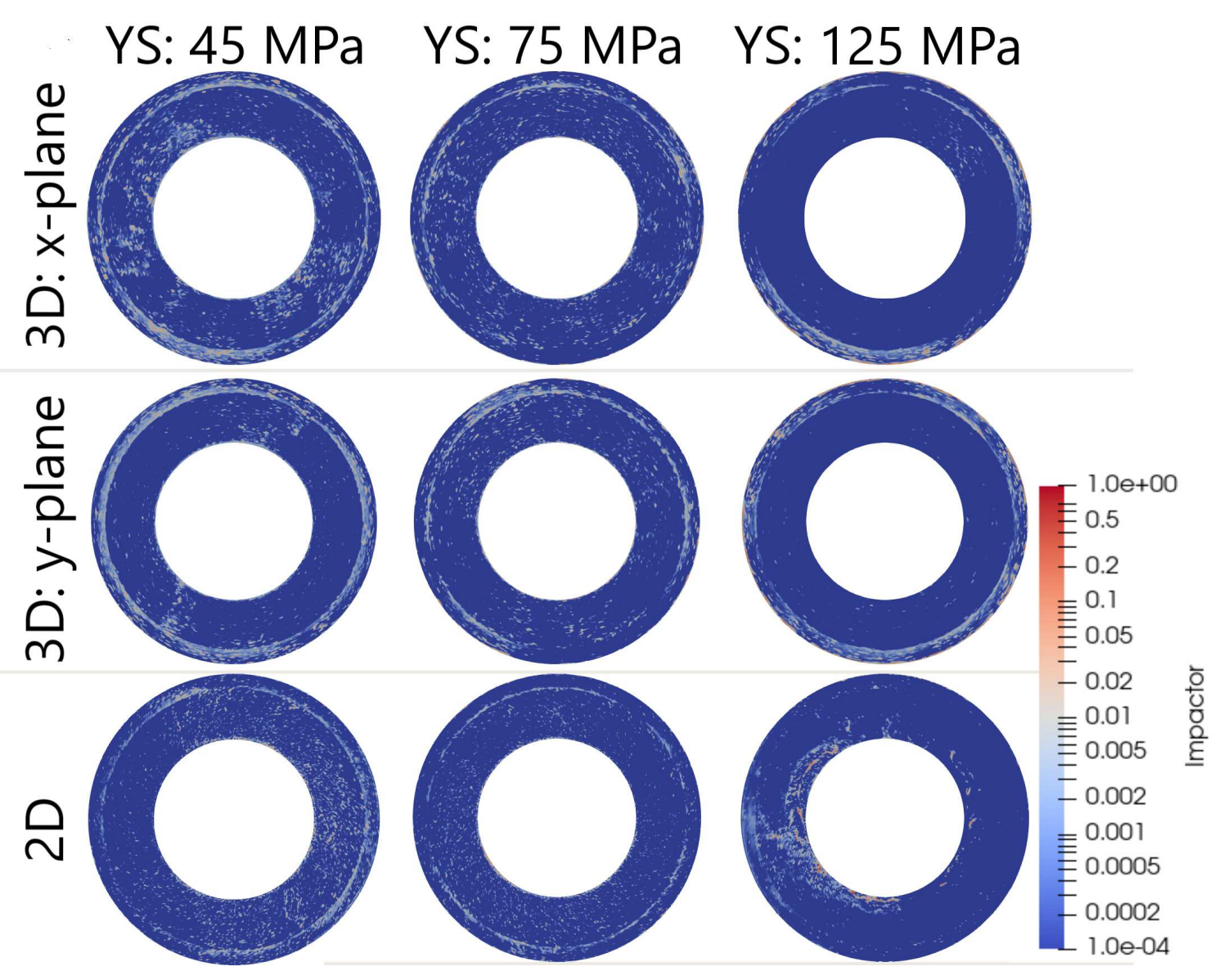

Figure 13 Slices of the radial distribution of impactor material at the end of simulations with impacts in 2-D and 3-D and for three different yield stress values. In 3D, two perpendicular pole-to-pole slices are plotted 

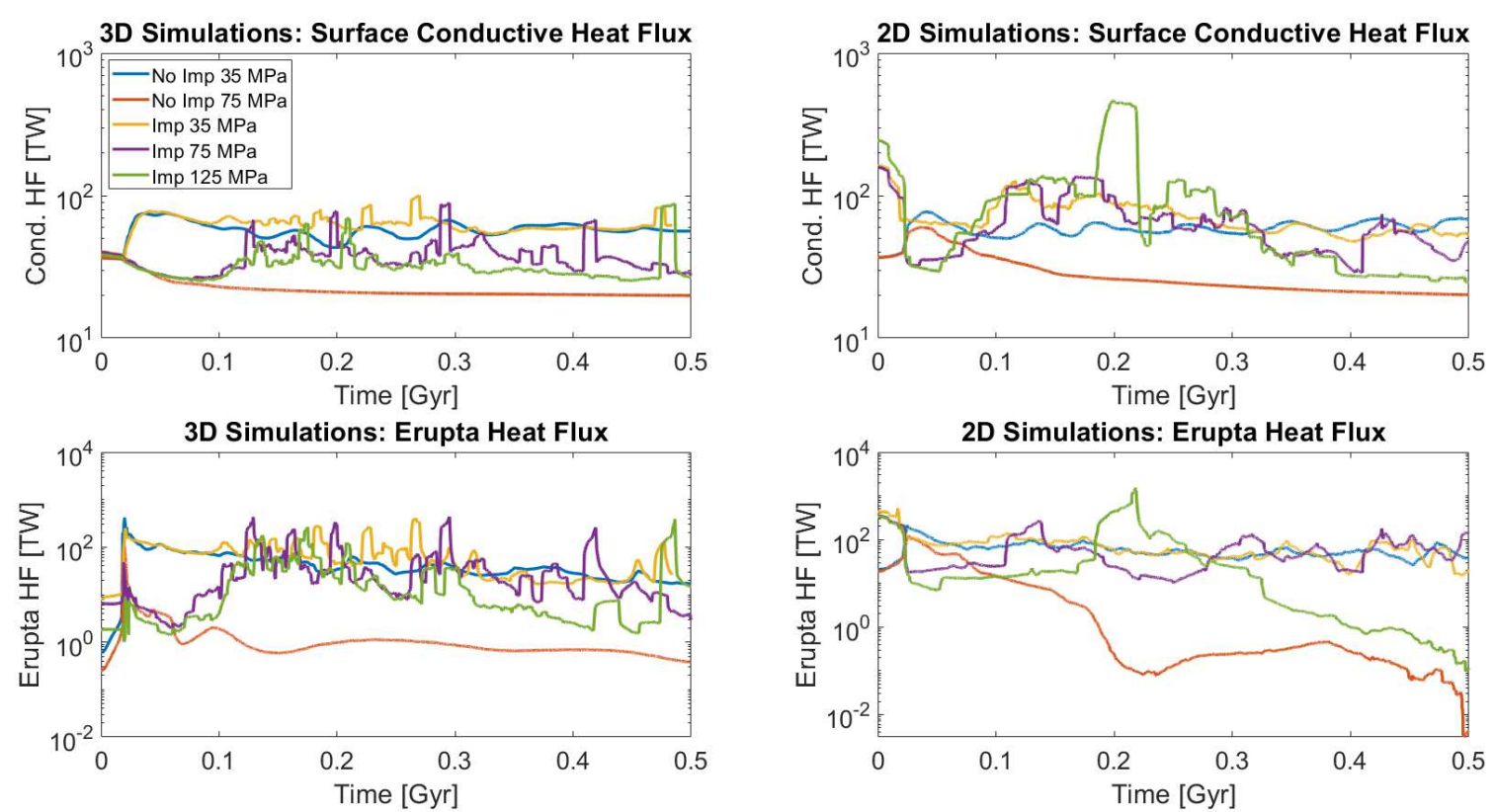

Figure 14 Time-evolution of conductive and eruptive surface heat fluxes for impact and no-impacts cases in 2-D and 3-D with various yield stresses. The upper panels show surface conductive heat flux while the lower panels show the eruptive heat flux. 


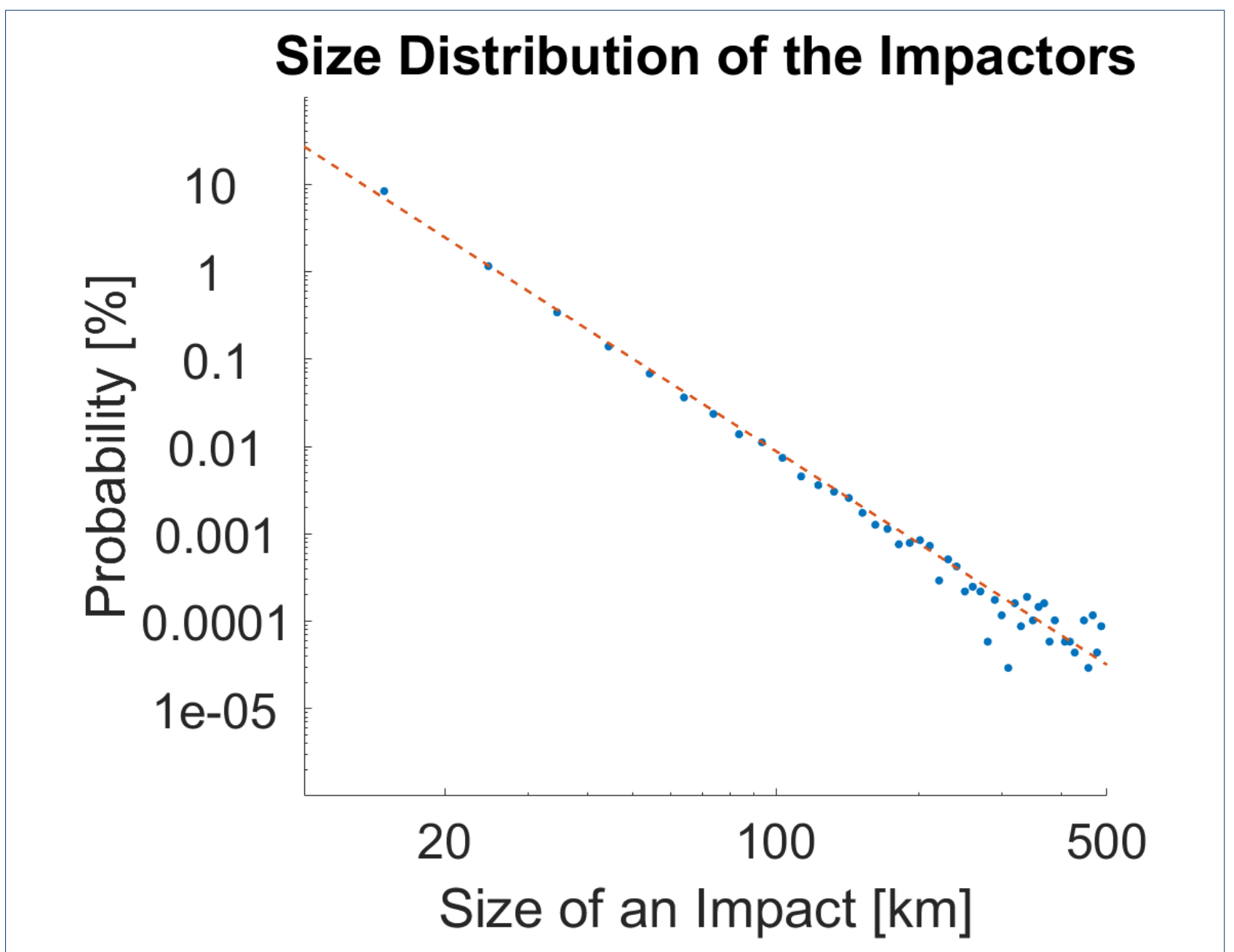

Figure 15 Normalized histogram of the size of impactors. Each point of the histogram corresponds to a bin spanning $10 \mathrm{~km}$ in size. This histogram contains impactors of 30 different simulations. The orange dashed curved has a slope of $10^{-3.5}$. 


\section{Supplementary Files}

This is a list of supplementary files associated with this preprint. Click to download.

- Animation1.mp4

- Animation2.mp4

- Abstractlmage.png

- pepsarticle.bib

- pepsart.bst

- pepsart.cls 